\title{
SIMPLICITY IN COMPACT ABSTRACT THEORIES
}

\author{
ITAY BEN-YAACOV
}

\begin{abstract}
We continue [Ben03], developing simplicity in the framework of compact abstract theories. Due to the generality of the context we need to introduce definitions which differ somewhat from the ones use in first order theories.

With these modified tools we obtain more or less classical behaviour: simplicity is characterised by the existence of a certain notion of independence, stability is characterised by simplicity and bounded multiplicity, and hyperimaginary canonical bases exist.
\end{abstract}

\section{INTRODUCTION}

Having defined the framework of compact abstract theories in [Ben03], one turns to develop tools.

The development of simplicity in [Pil00] extends quite well to cats, and many of the proofs here are taken from this paper. However, the definition of simplicity in [Pil00] raises a few problems (present already in the e.c. case). There, simplicity is defined by the following two properties:

(i) Dividing satisfies the local character.

(ii) Morley sequences exist in every type.

In the case of a general cat, the first does not necessarily imply the second (see Example 4.3). Moreover, it would seem on closer inspection that these two properties cannot be given equal status:

The first property is robust, in the sense that in order to know that it holds we do not need to verify it for all types, but only for "sufficiently many". More explicitly, we prove below that the local character of dividing is equivalent to the existence of an automorphism-invariant co-final class $\mathcal{A}$ of sets (that is, for every set $A$ there is $B$ such that $A \subseteq B \in \mathcal{A}$ ), and of a regular cardinal $\kappa>|T|$, such that for every increasing sequence $\left(A_{i} \in \mathcal{A}: i<\kappa\right)$, a type over $\bigcup A_{i}$ does not divide over some $A_{i}$. This implies, for example, that the local character is preserved when adjoining new hyperimaginary sorts (this is important: after all, the ability to adjoin hyperimaginary sorts as real elements was at the origin of the definition of cats).

On the other hand, even assuming that Morley sequences exist for types over certain domains, we do not know whether this implies that Morley sequences exist for types over other sets (except for a few particular cases). In particular, even if we assume that every type over a set of real elements has a Morley sequence, this does not mean we know that every type over a hyperimaginary domain has one.

Date: December 5, 2003.

2000 Mathematics Subject Classification. 03C95, 03C45.

At the time of the writing of this paper, the author was a graduate student with the Équipe de Logique Mathématique of Université Paris VII. 
Moreover, we would like stability to imply simplicity: stability implies the local character of dividing, but it does not imply that Morley sequences always exist. And of course, we know that in first order theories, the local character suffices for the development of simplicity.

In this paper we show that with some additional technical effort, simplicity can be developed from the local character alone, without the existence of Morley sequences as an additional assumption. In fact, we prove the symmetry and transitivity of dividing, as well as its characterisation by equality of local $D$-ranks, without ever mentioning Morley sequences. This gives, even in the first order case, a new approach to the first steps of the development of simplicity theory, essentially different from the classical one.

In order to do this, we introduce the somewhat technical notion of array-dividing. We prove that simplicity (i.e., the local character of dividing) implies the local character of array-dividing, and use it to prove that array-dividing is symmetric and transitive, and is characterised by equality of appropriate local $D$-ranks. Only then can we prove that in fact, array-dividing defines the same independence relation as dividing: we may then forget about this little detour, and proceed with the classical definitions.

We obtain that dividing independence satisfies all the usual axioms but extension and the independence theorem. For extendible type, that is types that satisfy the extension axiom, all the axioms hold, and we show that there are enough of these.

We conclude by showing that stability is equivalent to simplicity with bounded multiplicity of types, and that extendible amalgamation bases have hyperimaginary canonical bases, which can be adjoined to the structure in a new sort.

In order to put things in their proper context one should also mention [BL03], although it is independent of the present paper. There simplicity is developed for big homogeneous structures (equivalently: an abstract elementary class with amalgamation and type-locality). In very big lines, it has the advantage of having a more general context (no compactness is required), and the disadvantage of more complicated definitions and somewhat weaker results (in particular, canonical bases are not hyperimaginaries, do not satisfy type-locality, and therefore do not find their place in a homogeneous finitary structure).

On the other hand, there has been significant progress in the study of independence theory in cats with additional hypotheses. First, in [Bena], we prove that in order to get the full power of simplicity theory (as in first order theories), it suffices to assume thickness, namely that indiscernibility is a type-definable property. A stronger hypothesis than thickness is being Hausdorff, defined in [Ben03] as the property of having Hausdorff type-spaces. In [Benb] it is shown that Hausdorff cats admit a typedefinable metric on their universal domains, which is unique up to uniform equivalence. This metric is required for the development of useful notions of supersimplicity and superstability, along lines similar to those Henson and Iovino followed in the case of stable Banach spaces. 


\section{Simple CATS}

We develop independence theory for simple cats, namely cats where dividing satisfies the local character (but not necessarily the extension axiom). In order to do this we define the tool of array-dividing.

We prove that simplicity is equivalent to the existence of an independence relation satisfying slight variants of the classical axioms, which is necessarily given by nondividing.

1.1. Dividing and ranks. We shall need a generalised notion of dividing. We recall first the notion of indiscernible array:

Definition 1.1. (i) An $\alpha$-dimensional array (or simply an $\alpha$-array) is a set $\left(b_{\sigma}\right.$ : $\left.\sigma \in \beta^{\alpha}\right)$.

(ii) Let $f_{i}: \gamma \hookrightarrow \beta$ be monotonous inclusions for $i<\alpha$. Define $\tilde{f}: \gamma^{\alpha} \rightarrow \beta^{\alpha}$ by $\tilde{f}(\sigma)(i)=f_{i}(\sigma(i))$ for $\sigma \in \gamma^{\alpha}$ and $i<\alpha$. Then $\left(b_{\tilde{f}(\sigma)}: \sigma \in \gamma^{\alpha}\right)$ is the $\gamma^{\alpha}$-sub-array of $\left(b_{\sigma}: \sigma \in \beta^{\alpha}\right)$ given by $\bar{f}$.

(iii) An array $\left(b_{\sigma}: \sigma \in \beta^{\alpha}\right)$ is $c$-indiscernible if $\beta \geq \omega$ and for every $n<\omega$, all the $n^{\alpha}$-sub-arrays have the same type over $c$.

A sequence $\left(b_{i}: i<\alpha\right)$ is $c$-indiscernible if it is such as a 1-dimensional array.

In this section we shall extract indiscernible sequences not once, as this is in the essence of the theory of dividing. Since Ramsey's theorem will fail us, we shall need a stronger tool, which has already become standard:

Lemma 1.2. Let $A$ be a set of parameters, and $\lambda \geq\left|\mathrm{S}_{\kappa}(A)\right|$ (for example, $\lambda=$ $\left.2^{|T|+|A|+\kappa}\right)$. Set $\mu=\beth_{\lambda^{+}}$. Then for any sequence $\left(a_{i}: i<\mu\right)$ of $\kappa$-tuples there is an $A$ indiscernible sequence $\left(b_{i}: i<\omega\right)$ such that for all $n<\omega$ there are $i_{0}<\ldots<i_{n-1}<\mu$ for which $\operatorname{tp}\left(b_{0} \ldots b_{n-1} / A\right)=\operatorname{tp}\left(a_{i_{0}} \ldots a_{i_{n-1}} / A\right)$.

Proof. As all chains of references for this lemma seem to end up somewhere in the mists of the past, we give the complete proof.

We construct by induction a sequence of types $p_{n}$, each one a complete $n \times \kappa$-type over $A$, such that for all $n$ :

(i) For any $i_{0}<\ldots<i_{m-1}<n$ we have $p_{n}\left(x_{0}, \ldots, x_{n-1}\right) \vDash p_{m}\left(x_{i_{0}}, \ldots, x_{i_{m-1}}\right)$.

(ii) For all $\eta<\mu$ there is $I \subseteq \mu,|I|=\eta$ such that every $n$ elements in order from $a_{I}$ satisfy $p_{n}$.

For $n=0$ there is nothing to do. Given $p_{n}$, consider the set $S$ of all $(n+1) \times \kappa$-types over $A$ that satisfy the first condition. If there is $q \in S$ that also satisfies the second, we are done. If not, then for each $q \in S$ there is an $\eta_{q}<\mu$ that witnesses it. As $|S| \leq \lambda<\operatorname{cf}(\mu)=\lambda^{+}$, we have that $\eta=\lambda+\sup \left\{\eta_{q}: q \in S\right\}<\mu$ is such that for all $q \in S$, for all $I \subseteq \mu$ with $|I|=\eta$, not all $(n+1)$-sub-tuples in order from $a_{I}$ satisfy $q$. As $\eta<\mu$, then $\eta<\beth_{\theta}$ for some $\theta<\lambda^{+}$. Write $\nu=\beth_{\theta+n+1}$. Then on the one hand, $\nu<\mu$. On the other, $\nu \geq \beth_{n}(\eta)^{+}$. By the induction hypothesis there is $I \subseteq \mu,|I|=\nu$ such that all $n$-tuples in order in $a_{I}$ satisfy $p_{n}$. As there are at most $\lambda$ possible $A$-types for $(n+1)$-tuples and $\lambda \leq \eta$, the Erdős-Rado theorem gives us $I^{\prime} \subseteq I$ with $\left|I^{\prime}\right|=\eta^{+}$ in which all $(n+1)$-tuples in order have the same type over $A$. This gives the wanted contradiction, and concludes the construction. Taking $p_{\omega}$ as the limit of the $p_{n}$ gives the type of the wanted sequence.

$\mathrm{QED}_{1.2}$ 
For finite-dimensional arrays, we have a criterion for indiscernibility that uses indiscernibility of sequences, and an analogue of Lemma 1.2:

Lemma 1.3. Let $l<\omega$.

(i) Consider an array $\left(b_{\sigma}: \sigma \in \alpha^{l}\right)$, and for $i<l, j<\alpha$ let $\bar{b}^{i, j}=\left(b_{\sigma}: \sigma \in\right.$ $\alpha^{l}, \sigma(i)=j$ ) (that is, the $j$ th hyperplane in the ith direction). Then $\left(b_{\sigma}\right)$ is $c$-indiscernible if and only if for every $i<l$, the sequence $\left(\bar{b}^{i, j}: j<\alpha\right)$ is $c$-indiscernible in the ordinary sense.

(ii) For any $c$ and $\kappa$ there is $\lambda$ such that if $\left(b_{\sigma}: \sigma \in \lambda^{l}\right)$ is a finite-dimensional array and $\left|b_{\sigma}\right| \leq \kappa$ for every $\sigma$, then there is a c-indiscernible array $\left(b_{\sigma}^{\prime}: \sigma \in \omega^{l}\right)$ such that for every $k<\omega$, every $k^{l}$-sub-array of $\left(b_{\sigma}^{\prime}: \sigma \in \omega^{l}\right)$ has the same type over $c$ as some $k^{l}$-sub-array of the original array.

Proof. (i) Clear.

(ii) Fix $c$ and $\kappa$, and set $\lambda_{0}=\kappa$. By induction on $i$, take $\lambda_{i+1}$ as given by Lemma 1.2 for sequences of tuples of length $\lambda_{i}$. Then $\lambda_{l}$ should do, and $\lambda_{\omega}=\sup _{i<\omega} \lambda_{i}$ works independently of $l$.

$\mathrm{QED}_{1.3}$

Definition 1.4. Here $\alpha$ is any ordinal, and $l, k<\omega$.

(i) A partial type $p(x, b) \alpha$-array-divides over $a$ if there is an $\alpha$-dimensional $a$ indiscernible array $\left(b_{\sigma}: \sigma \in \omega^{\alpha}\right)$ in $\operatorname{tp}(b / a)$ such that $\bigcup p\left(x, b_{\sigma}\right)$ is inconsistent. It divides over $a$ if it 1-array-divides over $a$.

It array-divides over $a$ if it $\alpha$-array-divides over $a$ for some $\alpha$.

(ii) Let $\varphi(x, y)$ be a formula. Let $\bar{y}=y_{0} \ldots y_{k-1}$. We say that $\psi(\bar{y})$ is a $k$ inconsistency witness for $\varphi$ if $T \vdash \neg\left(\psi(\bar{y}) \wedge \bigwedge_{i<k} \varphi\left(x, y_{i}\right)\right)$.

(iii) Let $\psi\left(y_{<k^{l}}\right)$ be any formula. Then $\tilde{\psi}^{l}\left(y_{\in \omega^{l}}\right)$ is the partial type saying that every $k^{l}$-sub-array of $\left(y_{\sigma}: \sigma \in \omega^{l}\right)$ satisfies $\psi$ (the value of $k$ can be deduced from $\psi$ and $l$ ).

(iv) If $\psi$ is a $k^{l}$-inconsistency witness for $\varphi(x, y)$, then an instance $\varphi(x, b) l$-arraydivides over a with respect to $\psi$, if there is an array $\left(b_{\sigma}: \sigma \in \omega^{l}\right)$ in $\operatorname{tp}(b / a)$ satisfying $\tilde{\psi}^{l}$.

Remark 1.5. To our knowledge, indiscernible arrays were first introduced in [Kim98], where it is proved that in a first order simple theory, dividing coincides with $l$-arraydividing for every $l<\omega$.

Lemma 1.6. For a partial type $p(x, b)$, the following are equivalent:

(i) $p$ l-array-divides over a for some finite $l \leq \alpha$.

(ii) $p \beta$-array-divides over a for some $\beta \leq \alpha$.

(iii) $p \alpha$-array-divides over a.

(iv) There are $k<\omega$, a finite $l \leq \alpha$, a formula $\varphi(x, y)$ such that $p(x, y) \vdash \varphi(x, y)$, and a $k^{l}$-inconsistency witness $\psi$ for $\varphi$, such that $\varphi(x, b) l$-array-divides over a with respect to $\psi$.

Proof. $\quad($ i $) \Longrightarrow$ (ii)., (ii) $\Longrightarrow$ (iii). Clear.

(iii) $\Longrightarrow\left(\right.$ iv). We assume that $p(x, b) \alpha$-array-divides over $a$, and let $\left(b_{\sigma}: \sigma \in \omega^{\alpha}\right)$ witness this. This mean that $\bigcup_{\sigma} p\left(x, b_{\sigma}\right)$ is inconsistent, so by compactness 
there is some $\varphi$ such that $p(x, y) \vdash \varphi(x, y)$, a finite $I \subseteq \omega^{\alpha}$, say $|I|=k$, and an $k$-inconsistency witness $\psi$ for $\varphi$ such that $\vDash \psi\left(b_{\in I}\right)$. By the indiscernibility of $\left(b_{\sigma}\right)$, we may assume that in fact $I \subseteq k^{\alpha}$. We are going to replace the dimension $\alpha$ (which may be infinite) with the set $F=k^{I}$, which is finite, of cardinality $k^{k}$. For $i<\alpha$ define $f_{i} \in F$ by $f_{i}(\sigma)=\sigma(i)$, and define $g: \alpha \rightarrow F$ by $g(i)=f_{i}$. For every $\sigma \in I$ define $\hat{\sigma} \in k^{F}$ by $\hat{\sigma}(f)=f(\sigma)$ for every $f \in F$. Then $(\hat{\sigma} \circ g)(i)=\hat{\sigma}\left(f_{i}\right)=f_{i}(\sigma)=\sigma(i)$ for every $i<\alpha$, and $\hat{\sigma} \circ g=\sigma$. Let $\hat{I}=\{\hat{\sigma}: \sigma \in I\} \subseteq k^{F}$, so $\hat{I} \circ g=I$. Let $y_{\in k^{F}}=\left(y_{\tau}: \tau \in k^{F}\right)$ be an array of variables in the sort of $y$. Since $\hat{I} \subseteq k^{F}$, we can define $\hat{\psi}\left(y_{\in k^{F}}\right)=\psi\left(y_{\in \hat{I}}\right)$. Then one easily verifies that $\left(b_{\sigma \circ g}: \sigma \in \omega^{F}\right)$ is an $a$-indiscernible array of dimension $|F|, \hat{\psi}$ is a $k^{F}$-inconsistency witness for $\varphi$, and $\vDash \hat{\psi}\left(b_{k^{F} \circ g}\right)$. This proves what we wanted.

Note that when $\psi$ is a $k$-inconsistency witness, $\hat{\psi}$ can be as much as a $k^{k^{k}}$ inconsistency witness.

(iv) $\Longrightarrow($ i). We obtain an indiscernible array by Lemma 1.3.

$\mathrm{QED}_{1.6}$

Corollary 1.7. $p$ array-divides over a if and only if it l-array-divides over a for some $l<\omega$.

So we may restrict ourselves to $l$-array-dividing for finite $l$ : this is particularly reassuring, as Lemma 1.3 only speaks about finite-dimensional arrays.

The following is easy:

Lemma 1.8. $\operatorname{tp}(a / b)$ does not $\alpha$-array-divides over $c$ if and only if for every $\alpha$ dimensional c-indiscernible array $b_{i}$ containing $b$ there is a bc-automorphism rendering it ac-indiscernible.

Corollary 1.9. The following hold in any theory:

Monotonicity: If $\operatorname{tp}\left(a a^{\prime} / b b^{\prime} c\right)$ does not $\alpha$-array-divide over $c$, then neither does $\operatorname{tp}(a / b c)$.

Partial downward transitivity: If $\operatorname{tp}\left(a / b b^{\prime} c\right)$ does not $\alpha$-array-divide over $c$, then neither does it $\alpha$-array-divide over bc.

Partial upward transitivity: If $\operatorname{tp}(a / b c d)$ does not $\alpha$-array-divide over bc and $\operatorname{tp}(b / c d)$ does not $\alpha$-array-divide over $c$ then $\operatorname{tp}(a b / c d)$ does not $\alpha$-array-divide over $c$.

Proof. Monotonicity and partial downward transitivity are clear. For the partial upward transitivity, we use the previous lemma. Let $\left(d_{\sigma}\right)$ be a $c$-indiscernible $\alpha$ dimensional array with $d_{0}=d$. As $\operatorname{tp}(b / c d)$ does not $\alpha$-array-divide over $c,\left(d_{\sigma}\right)$ has a $c d$-automorphic image that is $b c$-indiscernible. As $\operatorname{tp}(a / b c d)$ does not $\alpha$-array-divide over $b c$, a $b c d$-automorphism sends it to an $a b c$-indiscernible array. The composition is a $c d$-automorphism that shows that $\operatorname{tp}(a b / c d)$ does not $\alpha$-array-divide over $c$. $\operatorname{QED}_{1.9}$

We also define the $D(-, \varphi, \psi, l)$-rank:

Definition 1.10. For a partial types $p(x)$ (with parameters), a formula $\varphi(x, y), l<\omega$ and a $k^{l}$-inconsistency witness $\psi(\bar{y})$ for $\varphi$, we define $D(p, \varphi, \psi, l)$ as the minimal value in $\omega \cup\{-1, \infty\}$ that satisfies: 
- $D(p, \varphi, \psi, l) \geq 0$ if $p$ is consistent.

- $D(p, \varphi, \psi, l) \geq n+1$ (for $n \in \omega$ ) if there are $\left(b_{\sigma}: \sigma \in \omega^{l}\right.$ ), satisfying $\tilde{\psi}^{l}$, and $D\left(p \wedge \varphi\left(x, b_{\sigma}\right), \varphi, \psi, l\right) \geq n$ for all $\sigma \in \omega^{l}$.

If $l=1$ we may omit it.

Definition 1.11. For a formula $\varphi$, a $k^{l}$-dividing witness $\psi$ for $\varphi$, a partial type $p$ and an ordinal $\alpha$ define:

$$
\begin{aligned}
\Psi_{\alpha, p}^{\varphi, \psi, l}\left(x_{\in\left(\omega^{l}\right)^{\alpha}}, y_{\in} \bigcup_{\beta<\alpha}\left(\omega^{l}\right)^{\beta+1}\right)= & \bigwedge_{\eta \in\left(\omega^{l}\right)^{\alpha}, \beta<\alpha} \varphi\left(x_{\eta}, y_{\eta \uparrow_{\beta+1}}\right) \\
& \wedge \bigwedge_{\eta \in\left(\omega^{l}\right)^{\alpha}} p\left(x_{\eta}\right) \\
& \wedge \bigwedge_{\beta<\alpha, \sigma \in\left(\omega^{l}\right)^{\beta}} \tilde{\psi}^{l}\left(y_{\sigma, s}: s \in \omega^{l}\right)
\end{aligned}
$$

If $l=1$ we may omit it.

Lemma 1.12. Let $p$ be a partial type, say over $A, \alpha$ an ordinal, and $\varphi, \psi, l$ as above. Then the following are equivalent:

(i) $D(p, \varphi, \psi, l) \geq \alpha$

(ii) $\Psi_{\alpha, p}^{\varphi, \psi, l}$ is consistent.

(iii) There exist $b_{\in \alpha \times \omega^{l}}$, such that for every $i<\alpha,\left(b_{i, \sigma}: \sigma \in \omega^{l}\right)$ is an indiscernible l-array over $A b_{\in i \times \omega^{l}}$ satisfying $\tilde{\psi}^{l}$, and $p(x) \wedge \bigwedge_{i<\alpha} \varphi\left(x, b_{i, 0^{l}}\right)$ is consistent.

Proof. $\quad($ i $) \Longrightarrow$ (ii). One proves by induction on $n<\omega$ that if $D(p, \varphi, \psi, l) \geq n$ then $\Psi_{n, p}^{\varphi, \psi, l}$ is consistent. For $\alpha \geq \omega, \Psi_{\alpha, p}^{\varphi, \psi, l}$ is consistent by compactness.

(ii) $\Longrightarrow$ (iii). By compactness, we can replace the $\omega^{l}$-arrays in $\Psi_{\alpha, p}^{\varphi, \psi, l}$ with $\lambda^{l}$ arrays, for any $\lambda$. Applying Lemma $1.3 \alpha$ times, we can find a realisation $\vDash \Psi_{\alpha, p}^{\varphi, \psi, l}\left(a_{\in\left(\omega^{l}\right)^{\alpha}}, b_{\left.\in \bigcup_{\beta<\alpha}\left(\omega^{l}\right)^{\beta+1}\right)}\right.$ such that in addition, for every $\beta<\alpha$ and $\sigma \in\left(\omega^{l}\right)^{\beta}$, the $l$-array $\left(b_{\sigma, s}: s \in \omega^{l}\right)$ is indiscernible (as an array) over

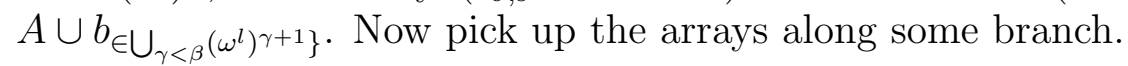

(iii) $\Longrightarrow$ (i). For $\alpha=n<\omega$, one proves by induction on $m \leq n$ that $D(p(x) \wedge$ $\left.\bigwedge_{i<n-m} \varphi\left(x, b_{i, 0^{l}}\right), \varphi, \psi, l\right) \geq m$, whereby $D(p, \varphi, \psi, l) \geq n$. For $\alpha \geq \omega$, the finite case implies $D(p, \varphi, \bar{\psi}, l)=\infty$.

$\mathrm{QED}_{1.12}$

Corollary 1.13. Let $p$ be a partial type over a set $A$. Then there is a complete type $q \in \mathrm{S}(A)$ extending $p$, such that $D(q, \varphi, \psi, l)=D(p, \varphi, \psi, l)$ ( $q$ depends on $\varphi, \psi, l)$.

Proof. By Lemma 1.12, there is $\alpha \leq \omega$ such that the value of $D(p, \varphi, \psi, l)$ is witnessed by the existence of $b_{\in \alpha \times \omega^{l}}$ such that for every $i<\alpha,\left(b_{i, \sigma}: \sigma \in \omega^{l}\right)$ is an indiscernible l-array over $A b_{\in i \times \omega^{l}}$ satisfying $\tilde{\psi}^{l}$, and $p(x) \wedge \bigwedge_{i<\alpha} \varphi\left(x, b_{i, 0^{l}}\right)$ is consistent. Choose $a$ such that $\vDash p(a) \wedge \bigwedge_{i<\alpha} \varphi\left(a, b_{i, 0^{l}}\right)$, and let $q=\operatorname{tp}(a / A)$. Then the same $b_{\in \alpha \times \omega^{l}}$ witness that $D(q, \varphi, \psi, l)=D(p, \varphi, \psi, l)$.

QED $_{1.13}$

Corollary 1.14. Let $p$ be a partial type, $p(x) \vdash \bigvee_{i<\lambda} \chi_{i}\left(x, b_{i}\right)$. Then $D(p, \varphi, \psi, l)=$ $\max D\left(p \wedge \chi_{i}\left(x, b_{i}\right), \varphi, \psi, l\right)$. In particular, the maximum is attained. 
Proof. Let $A$ contain all the parameters for $p$, as well as all the $b_{i}$, and let $q \in \mathrm{S}(A)$ extend $p$ such that $D(q, \varphi, \psi, l)=D(p, \varphi, \psi, l)$. Then $q \vdash p \wedge \chi_{i_{0}}\left(x, b_{i_{0}}\right)$ for some $i_{0}<\lambda$, so $D(p, \varphi, \psi, l)=D(q, \varphi, \psi, l) \leq D\left(p \wedge \chi_{i_{0}}\left(x, b_{i_{0}}\right), \varphi, \psi, l\right) \leq \sup D\left(p \wedge \chi_{i}\left(x, b_{i}\right), \varphi, \psi, l\right) \leq$ $D(p, \varphi, \psi, l)$, so there is equality all the way and the supremum is in fact a maximum.

$\mathrm{QED}_{1.14}$

This is clear:

Fact 1.15. Let $p$ be a partial type over $A$, and $q \supseteq p$ l-array-dividing over $A$. Then there are $\varphi, \psi$ such that either $D(q, \varphi, \psi, l)<D(p, \varphi, \psi, l)$ or $D(q, \varphi, \psi, l)=\infty$.

And we obtain the robustness of the local character:

Definition 1.16. A co-final class is a class $\mathcal{A}$ of subsets of the universal model $U$, invariant under automorphisms. We may alternatively consider it as a class of (infinite) tuples, where a tuple is in $\mathcal{A}$ if it enumerates a set in $\mathcal{A}$ (this is because we prefer enumerated tuples to sets in general).

Proposition 1.17. For a cat $T$ and $l<\omega$, the following are equivalent:

(i) $D(x=x, \varphi, \psi, l)<\infty$ for all $\varphi(x, y)$ and $k^{l}$-inconsistency witness $\psi\left(y_{\in k^{l}}\right.$ for $\varphi$.

(ii) l-array-dividing satisfies a strong local character: For any set $A$ and possibly infinite tuple $b$, there is $A_{0} \subseteq A$ with $\left|A_{0}\right| \leq|T|+|b|$, such that $\operatorname{tp}(b / A)$ does not l-array-divide over $A_{0}$.

(iii) l-array-dividing satisfies a weak local character: There is some $\lambda$, such that for any set $A$ and finite tuple $b$, there is $A_{0} \subseteq A$ with $\left|A_{0}\right| \leq \lambda$, such that $\operatorname{tp}(b / A)$ does not l-array-divide over $A_{0}$.

(iv) For any co-final class $\mathcal{A}, \mathcal{A}$-l-array-dividing satisfies a strong local character: For any possibly infinite tuple $b$, and for any increasing sequence $\left(A_{i}: i<\mu\right)$ of $\mathcal{A}$-sets such that $\mathrm{cf}(\mu)>|T|+|b|$, there is some $j<\mu$ such that $\operatorname{tp}\left(b / A_{<\mu}\right)$ does not l-array-divide over $A_{j}$.

(v) For some co-final class $\mathcal{A}, \mathcal{A}$-l-array-dividing satisfies a weak local character: There is some $\mu$, such that for any finite tuple $b$, and for any increasing sequence $\left(A_{i}: i<\mu\right)$ of $\mathcal{A}$-sets, there is some $j<\mu$ such that $\operatorname{tp}\left(b / A_{<\mu}\right)$ does not l-array-divide over $A_{j}$.

Proof. $\quad(\mathrm{i}) \Longrightarrow$ (ii). Take a subset $A_{0} \subseteq A$ such that $D(\operatorname{tp}(b / A), \varphi, \psi, l)=$ $D\left(\operatorname{tp}\left(b / A_{0}\right), \varphi, \psi, l\right)$ for all $\varphi, k<\omega$, and $k^{l}$-inconsistency witness $\psi$. $A_{0}$ can be taken to be of cardinality at most $|T|+|b|$, as there are at most $|T|+|b|$ $D(-, \varphi, \psi, l)$-ranks to be considered. By Fact $1.15, \operatorname{tp}(b / A)$ does not $l$-arraydivide over $A_{0}$.

(ii) $\Longrightarrow$ (iii)., (iv) $\Longrightarrow$ (v). Clear.

(ii) $\Longrightarrow$ (iv). $\operatorname{tp}\left(b / A_{<\mu}\right)$ does not $l$-array-divide over some subset of cardinality at most $|T|+|b|$, which is contained in some $A_{j}$ as $\operatorname{cf}(\mu)>|T|+|b|$.

(iii) $\Longrightarrow$ (v). Similarly, and take $\mu=\lambda^{+}$.

(v) $\Longrightarrow(\mathrm{i})$. Assume that $D(x=x, \varphi, \psi, l)=\infty$. By Lemma 1.12 we obtain a sequence $\left(b_{i}: i<\mu\right)$ of arbitrary length, such that $\bigwedge_{i<\mu} \varphi\left(x, b_{i}\right)$ is consistent, and $\varphi\left(x, b_{i}\right) l$-array-divides over $b_{<i}$, as witnessed by $\left(b_{i}^{\sigma}: \sigma \in \omega^{l}\right)$. Write $q(Y)=q\left(y_{<\mu}\right)=\operatorname{tp}\left(b_{<\mu}\right)$, and for $i<\mu$ : $q_{i}=\operatorname{tp}\left(b_{<i}\right)$. We propose to 
construct by induction an increasing sequence $\left(A_{i}: i<\mu\right)$ of $\mathcal{A}$-sets, and a sequence $\left(c_{i}: i<\mu\right)$ of elements, realizing $q$, such that $c_{i} \in A_{i+1}$, and $\varphi\left(x, c_{i}\right) l$-array-divides over $A_{i}$. Indeed, suppose we have done so for all $j<i$, so $c_{<i} \vDash q_{i}$. Take some $A_{i} \in \mathcal{A}$ containing $A_{<i} c_{<i}$, and realize $q_{i+1}\left(c_{<i}, y_{i}\right)$ in some $d$. By choice of $q$, there is a $c_{<i}$-indiscernible array $\left(d^{\sigma}: \sigma \in \omega^{l}\right)$ containing $d$, such that $\left\{\varphi\left(x, d^{\sigma}\right)\right\}$ is inconsistent. Applying Lemma 1.3 we obtain such a sequence which is also $A_{i}$-indiscernible. Taking $c_{i}$ to be any element of this sequence gives the wanted properties, and the construction is complete. Now any realisation of $\bigwedge \varphi\left(x, c_{i}\right)$ contradicts the local character of $\mathcal{A}$-l-array-dividing.

$\mathrm{QED}_{1.17}$

Remark 1.18. It is in Proposition 1.17 that we find the robustness of the local character. In particular, it is preserved when adjoining new hyperimaginary sorts, as co-final classes remain co-final.

Definition 1.19. A cat $T$ is simple if dividing satisfies the local character (that is to say that any of the equivalent conditions of Proposition 1.17 is satisfied for $l=1$ ).

As we said above, our proof that simplicity implies symmetry and transitivity of dividing uses array dividing, and the corresponding $D(-, \varphi, \psi, l)$-ranks, instead of the Morley sequences used in the classical approach. In particular, we want these ranks to be finite, so we need:

Proposition 1.20. If $T$ is simple then l-array-dividing satisfies the local character for every $l<\omega$.

Proof. We prove that $l$-array-dividing satisfies the local character by induction on $l$. For $l=1$ this is the assumption, so assume for $l$, and prove for $l+1$ :

If not, then there are a formula $\varphi(x, y)$, numbers $k, l<\omega$, and a $k^{l+1}$-inconsistency witness $\psi$ for $\varphi$, such that $D(x=x, \varphi, \psi, l+1)=\infty$. By Lemma 1.12, for every cardinal $\mu$ there exist $b_{\in \mu \times \omega^{l+1}}$, such that for every $i<\mu,\left(b_{i, s}: s \in \omega^{l+1}\right)$ is an indiscernible $(l+1)$-array over $b_{\in i \times \omega^{l+1}}$ satisfying $\tilde{\psi}^{l+1}$, and $\bigwedge_{i<\mu} \varphi\left(x, b_{i, 0^{l+1}}\right)$ is consistent. Moreover, as we can choose $\mu$ arbitrarily great, we may assume that $\left(b_{\in\{i\} \times \omega^{l+1}}: i<\mu\right)$ is indiscernible.

Let $\mu=|T|^{+}$, and for $I \subseteq \mu$ write $q_{I}(x)=\bigwedge_{i \in I, s \in \omega^{l}} \varphi\left(x, b_{i, s, 0}\right) \wedge \bigwedge_{i \notin I} \varphi\left(x, b_{i, 0^{l+1}}\right)$. There are two possibilities:

- Either $q_{\mu}$ is consistent, in which case it divides over every $b_{\in i \times \omega^{l+1}}$ for every $i$, contradicting simplicity.

- Or not, in which case there is some $i_{0}<\mu$ such that $q_{i_{0}}$ is consistent, but $q_{i_{0}+1}$ is not. By indiscernibility of $\left(b_{\in\{i\} \times \omega^{l+1}}: i<\mu\right), q_{i_{0} \cup\{i\}}$ is inconsistent for any $i_{0} \leq i<\mu$, which means that $q_{i_{0}} l$-array-divides over $b_{\in i \times \omega^{l+1}}$ for every $i<\mu$, contradicting the induction hypothesis.

This contradiction shows that $(l+1)$-array-dividing satisfies the local character and the induction step is done.

QED $_{1.20}$

Corollary 1.21. $T$ is simple if and only if array-dividing satisfies the local character.

Remark 1.22. Note that we used Proposition 1.17 in the proof of Proposition 1.20, so we could not have restricted Proposition 1.17 to dividing. 
1.2. Symmetry and transitivity. The whole point of introducing array-dividing is in that it enables us to prove:

Lemma 1.23. Let $a, b, c$ be possibly infinite tuples, $x, y, z$ the corresponding tuples of variables. Then the following conditions imply one another from top to bottom:

(i) For all $k, l<\omega, \varphi(y, x z)$ and $k^{l}$-inconsistency witness $\psi$ for $\varphi$ :

$$
D(\operatorname{tp}(b / a c), \varphi, \psi, l)=D(\operatorname{tp}(b / c), \varphi, \psi, l)<\infty
$$

(ii) $\operatorname{tp}(b / a c)$ does not array-divide over $c$.

(iii) For all $k, l<\omega, \varphi(x, w)$ and $k^{l}$-inconsistency witness $\psi$ for $\varphi$ :

$$
D(\operatorname{tp}(a / b c), \varphi, \psi, l)=D(\operatorname{tp}(a / c), \varphi, \psi, l)
$$

Proof. $\quad(\mathrm{i}) \Longrightarrow$ (ii). Just use Fact 1.15.

(ii) $\Longrightarrow($ iii). Assume that $D(\operatorname{tp}(a / c), \varphi, \psi, l) \geq n$, so we can find $\vDash$ $\Psi_{n, \mathrm{tp}(a / c)}^{\varphi, \psi, l}\left(a_{\in\left(\omega^{l}\right)^{n}}, d_{\in \bigcup_{\beta<n}\left(\omega^{l}\right)^{\beta+1}}\right)$. Then $\left(a_{\sigma}: \sigma \in\left(\omega^{l}\right)^{n}\right)$ is an $l n$-array in $\operatorname{tp}(a / c)$, so in particular finite dimensional: by compactness and Lemma 1.3, we may assume that it is $c$-indiscernible. Now use the assumption that $\operatorname{tp}(b / a c)$ does not array-divide over $c$ to obtain $b^{\prime}$ such that $a_{\sigma} b^{\prime} \equiv_{c} a b$ for all $\sigma \in \omega^{l n}$. Let $f$ be an automorphism of the universal domain sending $b^{\prime}$ to $b$ while fixing $c$ : then $\vDash \Psi_{n, \mathrm{tp}(a / b c)}^{\varphi, \psi, l}\left(f\left(a_{\in\left(\omega^{l}\right)^{n}}\right), f\left(d_{\in \bigcup_{\beta<n}\left(\omega^{l}\right)^{\beta+1}}\right)\right)$, whereby $D(\operatorname{tp}(a / b c), \varphi, \psi, l) \geq n$. The equality ensues.

$\mathrm{QED}_{1.23}$

The first implication actually holds for each value of $l$ separately: Fact 1.15 says precisely that for every $l$, if $D(\operatorname{tp}(b / a c), \varphi, \psi, l)=D(\operatorname{tp}(b / c), \varphi, \psi, l)<\infty$ for all $\varphi, \psi$, then $\operatorname{tp}(b / a c)$ does not $l$-array-divide over $c$.

On the other hand, in the second implication, the dimension of the arrays under consideration may increase arbitrarily: in order to prove that $D(\operatorname{tp}(a / b c), \varphi, \psi, l)=$ $D(\operatorname{tp}(a / c), \varphi, \psi, l)$ we need non- $n l$-array-dividing, and we have no bound for $n$ (even if $l$ is fixed, $D(x=x, \varphi, \psi, l)$ varies with $\varphi, \psi)$. Thus, even if we only want to prove that $D(\operatorname{tp}(a / b c), \varphi, \psi)=D(\operatorname{tp}(a / c), \varphi, \psi)$ for all $\varphi, \psi$, we need to assume that $\operatorname{tp}(b / a c)$ does not array-divide over $c$ : ordinary non-dividing does not, a priori, suffice. This should explain the need for the rather technical definitions and results above, and in particular for Proposition 1.20.

Convention 1.24. From here on we assume that $T$ is simple.

Notation 1.25. We write $a \downarrow_{c} b$ to say that $\operatorname{tp}(a / b c)$ does not array-divide over $c$.

Then Lemma 1.23 yields:

Corollary 1.26. The following are equivalent:

(i) $a \downarrow_{c} b$

(ii) $b \downarrow_{c} a$

(iii) $D(\operatorname{tp}(a / b c), \varphi, \psi, l)=D(\operatorname{tp}(a / c), \varphi, \psi, l)$ for every $\varphi, \psi, l$ for which this is defined.

(iv) $D(\operatorname{tp}(b / a c), \varphi, \psi, l)=D(\operatorname{tp}(b / c), \varphi, \psi, l)$ for every $\varphi, \psi, l$ for which this is defined. 
Corollary 1.27. Independence is symmetric and transitive:

(i) $a \downarrow_{c} b \Longleftrightarrow b \downarrow_{c} a$

(ii) $a \downarrow_{c}^{c} b d \Longleftrightarrow a \downarrow_{c} b \wedge a \downarrow_{b c} d$.

Proof. Symmetry was proved in Corollary 1.26. Transitivity follows either from symmetry and partial upward and downward transitivity, or from the characterisation of independence by equality of $D(-, \varphi, \psi, l)$-ranks.

QED $_{1.27}$

We defined simplicity by the local character of dividing, proved that the local character of array-dividing follows, and used it to prove that non-array-dividing is symmetric and transitive. We may now complete the cycle by proving:

Proposition 1.28. The following are equivalent:

(i) $a \downarrow_{c} b$.

(ii) $D(a / b c, \varphi, \psi)=D(a / c, \varphi, \psi)$ for every $\varphi, \psi$ for which this makes sense.

(iii) $\operatorname{tp}(a / b c)$ does not divide over $c$.

Proof. $\quad($ i $) \Longrightarrow$ (ii). Take $l=1$ in Corollary 1.26.

(ii) $\Longrightarrow$ (iii). Take $l=1$ in Fact 1.15 .

(iii) $\Longrightarrow$ (i). It would suffice to show that $D(b / a c, \varphi, \psi, l)=D(b / c, \varphi, \psi, l)$ for every $\varphi, \psi, l$.

So fix such a triplet: by Corollary 1.13 we can find a sequence $\left(b_{i}: i<|T|^{+}\right)$ such that $b_{i} \equiv_{c} b$ and $D\left(b_{i} / c b_{<i}, \varphi, \psi, l\right)=D(b / c, \varphi, \psi, l)$ for every $i$. By Lemma 1.2 we may assume that this sequence is $c$-indiscernible, and since $\operatorname{tp}(a / b c)$ does not divide over $c$ we may further assume that $b_{i} \equiv_{a c} b$ for all $i$. By the local character of array-dividing, there is $i<|T|^{+}$such $a \downarrow_{c b_{<i}} b_{i}$, whereby: $D(b / a c, \varphi, \psi, l)=D\left(b_{i} / a c, \varphi, \psi, l\right) \geq D\left(b_{i} / a c b_{<i}, \varphi, \psi, l\right)=$ $D\left(b_{i} / c b_{<i}, \varphi, \psi, l\right)=D(b / c, \varphi, \psi, l)$.

QED $_{1.28}$

Therefore, from this point onwards, we shall mention array-dividing no more.

1.3. Extension. By Example 4.3, the extension axiom need not always holds in a simple cat. Still, as the extension axiom plays an essential role in simplicity theory, we should like to distinguish those types for which it does hold. We characterise them as those types for which Morley sequences exist, and show that they these types are quite abundant.

Definition 1.29. A complete type $p \in \mathrm{S}(A)$ is called extendible if it has non-dividing extensions to every set $B \supseteq A$.

This notion is closely related to that of a Morley sequence:

Definition 1.30. A sequence $\left(a_{i}\right)$ is independent over $c$ if $a_{i} \downarrow_{c} a_{<i}$ for all $i$. If in addition it is $c$-indiscernible, then it is a Morley sequence over $c$. A Morley sequence over $c$ for $a$ is a Morley sequence $\left(a_{i}\right)$ over $c$, such that $a_{0}=a$.

Fact 1.31. If $\left(a_{i}: i \in I\right)$ is an independent set over $c$, then $a_{\in I^{\prime}} \downarrow_{c} a_{\in I^{\prime \prime}}$ for every disjoint $I^{\prime}, I^{\prime \prime} \subseteq I$. 
Proof. For finite $I^{\prime}, I^{\prime \prime}$, this is by symmetry and transitivity. For infinite ones, by the finite character.

QED $_{1.31}$

The usefulness of Morley sequences lies in the following property, first noticed in [Kim98]:

Proposition 1.32. Let $\left(a_{i}: i<\alpha\right)$ be a Morley sequence over $c$, and $b$ any tuple. Then:

(i) If $\operatorname{cf}(\alpha)>|T|+|b|$ then there is $i<\alpha$ such that $a_{\in[i, \alpha)} \downarrow_{c} b$.

(ii) If $\left(a_{i}\right)$ is bc-indiscernible, then $a_{<\alpha} \downarrow_{c} b$.

Proof. $\quad$ (i) By the local character there is $i<\alpha$ such that $b \downarrow_{a_{<i} c} a_{\in[i, \alpha)}$. Since this is a Morley sequence we also have $a_{<i} \downarrow_{c} a_{\in[i, \alpha)}$, so $a_{\in[i, \alpha)} \downarrow_{c} b$ by transitivity.

(ii) Since the sequence is $b c$-indiscernible we may extend it to length $(|T|+|b|)^{+}$, and apply the previous item and automorphism invariance.

$\mathrm{QED}_{1.32}$

The connection is given by:

Lemma 1.33. A type $p=\operatorname{tp}(a / c)$ is extendible if and only if it has a Morley sequence.

Proof. $\quad \Longrightarrow$ If $p$ is extendible we can construct a long $c$-independent sequence in $p$, and then apply Lemma 1.2.

$\Longleftarrow$ Let $\left(a_{i}\right)$ be a Morley sequence in $p$ over $c$, and let $b$ be any tuple. Assuming it is long enough we find some $a_{i} \downarrow_{c} b$ by Proposition 1.32, $\operatorname{sotp}\left(a_{i} / b c\right)$ is the required extension.

QED $_{1.33}$

We can show that extendible types exist:

Proposition 1.34. $\quad$ (i) Assume that $a \downarrow_{c} b$. Then $\operatorname{tp}(a / c)$ is extendible if and only if $\operatorname{tp}(a / b c)$ is.

(ii) Assume that $\operatorname{tp}(a / b c)$ and $\operatorname{tp}(b / c)$ are extendible. Then $\operatorname{tp}(a b / c)$ is.

(iii) Assume that $\operatorname{tp}(a b / c)$ is extendible. Then $\operatorname{tp}(a / c)$ is.

(iv) A type $\operatorname{tp}\left(a_{\in I} / c\right)$ is extendible if and only if $\operatorname{tp}\left(a_{\in I_{0}} / c\right)$ is extendible for every finite $I_{0} \subseteq I$. We could say that the property of being extendible has a finite character.

(v) Assume that $\left\{a_{i}: i \in I\right\}$ are c-independent, and $\operatorname{tp}\left(a_{i} / c\right)$ is extendible for all $i \in I$. Then $\operatorname{tp}\left(a_{\in I} / c\right)$ is extendible.

Proof. (i) Let $\left(a_{i}: i<\omega\right)$ be a Morley sequence for $a$ over $c$. As $a \downarrow_{c} b$, we may assume that it is indiscernible over $b c$ in $\operatorname{tp}(a / b c)$. Then $a_{<\omega} \downarrow_{c} b$, and $\left(a_{i}\right)$ is a Morley sequence over $b c$ by transitivity. Conversely, a Morley sequence for $a$ over $b c$ is also a Morley sequence over $c$, by transitivity.

(ii) Let $d$ be any tuple, and we want to find a non-dividing extension for $\operatorname{tp}(a b / c)$ to $d c$. First realize $\operatorname{tp}(b / c)$ by $b^{\prime} \downarrow_{c} d$, then find $a^{\prime}$ such that $a^{\prime} \downarrow_{b^{\prime} c} d$ and $a^{\prime} b^{\prime} \equiv_{c} a b$. Then $a^{\prime} b^{\prime} \downarrow_{c} d$ by transitivity.

(iii) Clear. 
(iv) Left to right is clear. We prove right to left by induction on $\kappa=|I|$, and we may in fact assume that $I=\kappa$. If $\kappa<\omega$ this is tautological, so we may assume that $\kappa \geq \omega$. In this case, $p_{i}=\operatorname{tp}\left(a_{<i} / c\right)$ is extendible for every $i<\kappa$ by the induction hypothesis.

Let $d$ be any tuple. We want to construct an increasing sequence of types $q_{i}$, each of which being a non-dividing extension of the corresponding $p_{i}$ to $c d$. At limits (and 0) there is no problem. For $i+1$ take a Morley sequence $\left(a_{\leq i}^{j}: j<\omega\right)$ in $\operatorname{tp}\left(a_{\leq i} / c\right)$. As $q_{i}$ does not divide over $c$, we may assume that $\bigwedge_{j} q_{i}\left(a_{<i}^{j}\right)$, and furthermore that $\left(a_{\leq i}^{j}: j<\omega\right)$ is $c d$-indiscernible. Then $a_{\leq i}^{0} \downarrow_{c} d$, so we can take $q_{i+1}=\operatorname{tp}\left(a_{\leq i}^{0} / c \bar{d}\right)$.

Thus $q=\bigcup q_{i}$ is a non-dividing extension of $\operatorname{tp}\left(a_{<\kappa} / c\right)$ to $c d$.

(v) For finite $I$ use (i) and (ii); for infinite $I$, use the finite case and (iv).

$\mathrm{QED}_{1.34}$

Proposition 1.35. There is a cardinal $\lambda$ such that every type over a $\lambda$-compact model is extendible.

Proof. Write $\lambda=\beth_{\left(2^{|T|}\right)+}$. We want to prove that if $M$ is $\lambda$-compact and $a$ is any tuple, then $\operatorname{tp}(a / M)$ is extendible.

By Lemma 1.34, we may assume that $a$ is finite. By the local character, there exists $A \subseteq M,|A| \leq|T|$ such that $a \downarrow_{A} M$. Using the fact that $M$ is $\lambda$-compact, we can construct a sequence $\left(a_{i}: i<\lambda\right)$ in $M$ such that $a_{i} \equiv_{A a_{<i}} a$ for every $i<\lambda$. Then $a \downarrow_{A} M \Longrightarrow a_{i} \downarrow_{A} a_{<i}$ for every $i$, and $\left(a_{i}\right)$ is an independent sequence over $A$. By Lemma 1.2 , we obtain a Morley sequence in $\operatorname{tp}(a / A)$, whereby $\operatorname{tp}(a / A)$ is extendible. Since $a \downarrow_{A} M$, we get that $\operatorname{tp}(a / M)$ is extendible as well. $\quad$ QED $_{1.35}$

Definition 1.36. Let $\mathcal{A}$ be a co-final class. Then $T$ is $\mathcal{A}$-simple if it is simple, and every type over an $\mathcal{A}$-set is extendible.

Thus, if $\lambda$ is as in Proposition 1.35 and $\mathcal{A}$ is the class of $\lambda$-compact models, then $\mathcal{A}$ is co-final and $T$ is $\mathcal{A}$-simple.

Question 1.37. Does $\lambda=|T|^{+}$suffice for Proposition 1.35?

1.4. The independence theorem. We pass to Lascar strong types and the independence theorem. We follow a path similar to [Sha00, Pil00], with some needed improvements.

Lemma 1.38. Let $a$ and $b$ be two tuples of the same sort, and $c$ any tuple. Then conditions (i) and (ii) below are equivalent. If $T$ is simple and $\operatorname{tp}(a / c)$ is extendible, then they are further equivalent to condition (iii):

(i) $a$ and $b$ are equivalent under every c-invariant bounded equivalence relation (that is, which has a bounded number of classes) on the sort of a.

(ii) There are $n<\omega$ and $a=a_{0}, \ldots, a_{n}=b$ such that for every $i<n, a_{i}, a_{i+1}$ are on some c-indiscernible sequence.

(iii) There are $a=a_{0}, \ldots, a_{n}=b$ such that every $a_{i}, a_{i+1}$ are on some Morley sequence over $c$. 
Proof. $\quad($ i $) \Longrightarrow$ (ii). The relation in (ii) is clearly a $c$-invariant equivalence relation, so we have to show it is bounded. If not, Lemma 1.2 would give a $c$-indiscernible sequence of non-equivalent elements, contradicting the definition.

(ii) $\Longrightarrow$ (i). Any two elements on a $c$-indiscernible sequence must clearly be equivalent by any bounded $c$-invariant equivalence relation.

(i) $\Longrightarrow$ (iii). We assume that $T$ is simple and $\operatorname{tp}(a / c)$ is extendible.

As above, the relation in (iii) is clearly a $c$-invariant equivalence relation, and we have to show that it is bounded. We may restrict it to the realisations of $p=\operatorname{tp}(a / c)$, saying that any two elements not satisfying $p$ are equivalent, so it suffices to prove that it has boundedly many classes on the realisations of $p$. Assume the contrary, and find for any $\kappa$ a $c$-independent sequence $\left(a_{i}: i<\kappa\right)$ of inequivalent realisations of $p$ : Having found $a_{<i}$ for $i<\kappa$, let $b \vDash p$ be inequivalent to all of $a_{<i}$, and let $\left(b_{j}\right)$ be a Morley sequence over $c$ with $b_{0}=$ $b$. Assuming it is long enough and applying Proposition 1.32, we find some $b_{j} \downarrow_{c} a_{<i}$ on this sequence, and as $b_{j}$ in on the same Morley sequence over $c$ as $b$, it is not equivalent to any of $a_{<i}$ either. Take $a_{i}=b_{j}$.

Doing this for $\kappa$ big enough and applying Lemma 1.2 we obtain a Morley sequence in $p$ whose elements are inequivalent, contradicting the definition.

(iii) $\Longrightarrow$ (ii). Clear.

QED $_{1.38}$

Definition 1.39. We say that $a \equiv_{c}^{\mathrm{Ls}} b$ if any of the conditions of Lemma 1.38 is true. It is clearly the finest bounded $c$-invariant equivalence relation on any sort.

We have extension for extendible Lascar strong types:

Proposition 1.40. If $\operatorname{tp}(a / c)$ is extendible, then for every $b$ there is $a^{\prime} \equiv_{c}^{\mathrm{Ls}}$ a such that $a \downarrow_{c} b$.

Proof. Take a long Morley sequence $\left(a_{i}\right)$ for $a$ over $c$. Then by Proposition 1.32 there is some $a_{i} \downarrow_{c} b$, and $a_{i} \equiv_{c}^{\mathrm{Ls}} a$.

$\mathrm{QED}_{1.40}$

We prove the independence theorem in several steps:

Lemma 1.41. Assume that $\left\{a, b_{0}, b_{1}\right\}$ is a c-independent set, $d \downarrow_{c} a b_{0}$ and $b_{0}, b_{1}$ are on some Morley sequence over $c$. Then there exists $d^{\prime} \equiv_{a c} d$ such that $d^{\prime} b_{1} \equiv_{c} d b_{0}$ and $d^{\prime} \downarrow_{c} a b_{1}$

Proof. We may assume that $\left(b_{i}: i<\omega\right)$ is a Morley sequence over $c$. As $a \downarrow_{c b_{0}} b_{1}$, we may assume that $\left(a b_{0} b_{i}: 0<i\right)$ is $c$-indiscernible. Take it to be long enough, and for each $i$ find $a_{i}$ such that $\left(a_{i} b_{i} b_{i^{\prime}}: i^{\prime}>i\right)$ be similar over $c$ to $\left(a b_{0} b_{i}: i>0\right)$. Apply Lemma 1.2 to get $\left(a_{i} b_{i}\right)$ to be $c$-indiscernible, without changing $b_{0}, b_{1}$. As for all $i<i^{\prime}$ : $a_{i} b_{i} b_{i^{\prime}} \equiv_{c} a b_{0} b_{1}$, we may also assume that $a_{0}=a$. As we know that $d \downarrow_{c} a b_{0}$, there is $d^{\prime} \equiv_{c a b_{0}} d$ such that $\left(a_{i} b_{i}\right)$ is $c d^{\prime}$-indiscernible. Then $d^{\prime} \equiv_{a c} d$ and $d^{\prime} b_{1} \equiv_{c} d^{\prime} b_{0} \equiv_{c} d b_{0}$. In addition, $\left(b_{i}: 0<i\right)$ is a Morley sequence over $c$, indiscernible over $a c d^{\prime}$, whereby $a d^{\prime} \downarrow_{c} b_{1}$ and $d^{\prime} \downarrow_{c} a b_{1}$.

QED $_{1.41}$

Lemma 1.42 (Weak independence theorem). Assume that $a_{0} \downarrow_{c} a_{1}, b_{i} \downarrow_{c} a_{i}, b_{0} \equiv_{c}^{\mathrm{Ls}}$ $b_{1}$ and $\operatorname{tp}\left(a_{i} / c\right), \operatorname{tp}\left(b_{i} / c\right)$ are all extendible. Then there is $b \downarrow_{c} a_{0} a_{1}$ with $b \equiv_{a_{i} c} b_{i}$. 
Proof. We might as well assume that $\left\{a_{0}, a_{1}, b_{0}, b_{1}\right\}$ is a $c$-independent set. As $b_{0} \equiv_{c}^{\mathrm{Ls}} b_{1}$, there are $b_{0}=d_{0}, \ldots, d_{n}=b_{1}$ such that $d_{i}, d_{i+1}$ are on the same Morley sequence over c. As $a_{0} \downarrow_{c} a_{1}$, we know that $\operatorname{tp}\left(a_{0} a_{1} / c\right)$ is extendible, whereby we may assume that $a_{0} a_{1} \downarrow_{c} \bar{d}$. Successive applications of Lemma 1.41 would give $a_{1}^{\prime} \equiv_{a_{0} c} a_{1}$ such that $a_{1}^{\prime} b_{0} \equiv_{c} a_{1} b_{1}$ and $a_{1}^{\prime} \downarrow_{c} a_{0} b_{0}$. Send $a_{1}^{\prime}$ back to $a_{1}$ fixing $a_{0} c$ and take $b$ to be the image of $b_{0}$. Then $b \downarrow_{c} a_{0} a_{1}$ and $b \equiv_{a_{i} c} b_{i}$.

$\mathrm{QED}_{1.42}$

Corollary 1.43. Assume that $\operatorname{tp}(a / c)$ is extendible. Then $a \equiv_{c}^{\mathrm{Ls}} b$ and $a \downarrow_{c} b$ if and only if $a, b$ lie on some Morley sequence over $c$.

Proof. One direction is clear. For the other, the classical proof works: Write $p=$ $\operatorname{tp}(a b / c)$, and obtain a $c$-independent sequence $\left(a_{i}\right)$ such that for every $i<j$ we have $p\left(a_{i} a_{j}\right)$ and $a_{i} \equiv_{c a_{<i}} a_{j}$. Start with $a_{0}=a, a_{1}=b$. For $i$ limit, just take $a_{i}$ to realize $\bigcup_{j<i} \operatorname{tp}\left(a_{j} / c a_{<j}\right)$. For $i+1 \geq 2$, send $a$ to $a_{i}$ over $c$ and take $a_{i+1}^{\prime}$ to be the image of $b$. As $p(x y) \vdash x \equiv_{c}^{\mathrm{Ls}} y$, we have $a_{i} \equiv_{c}^{\mathrm{Ls}} a_{i+1}^{\prime}$. On the other hand, $a_{i} \downarrow_{c} a_{<i}$ and $a_{i} \downarrow_{c} a_{i+1}^{\prime}$, so we can amalgamate $\operatorname{tp}\left(a_{i} / c a_{<i}\right)$ and $\operatorname{tp}\left(a_{i+1}^{\prime} / c a_{i}\right)$ and obtain $a_{i+1} \downarrow_{c} a_{\leq i}$ satisfying the requirements. Apply Lemma 1.2 to finish.

$\mathrm{QED}_{1.43}$

Corollary 1.44. Assume that $p=\operatorname{tp}(a / c)$ is extendible. Then $\equiv_{c}^{\mathrm{Ls}}$ restricted to $p$ is type-definable.

Proof. Let $q(x, y)$ be the type over $c$ of two elements from some Morley sequence in $p$. Then $q(x, y) \vdash p(x) \wedge p(y) \wedge x \downarrow_{c} y \wedge x \equiv_{c}^{\mathrm{Ls}} y$. We claim that $a \equiv_{c}^{\mathrm{Ls}} b$ if and only if there are $a=a_{0}, a_{1}, a_{2}, a_{3}=b$ such that $q\left(a_{i}, a_{i+1}\right)$, and this condition is clearly type-definable. One direction is clear. For the other, assume that $a \equiv_{c}^{\mathrm{Ls}} b$. Take $a_{1}$ such that $q\left(a, a_{1}\right)$ and $a_{1} \downarrow_{a c} b$. Then in particular $a_{1} \downarrow_{c} b$, and we can amalgamate $q\left(a_{1}, x\right)$ and $q(x, b)$ to obtain the desired $a_{2}$.

$\mathrm{QED}_{1.44}$

Remark 1.45. Unlike in simple first order theories, the relation $\equiv_{c}^{\mathrm{Ls}}$ is not necessarily type-definable. In fact, in a simple cat, type-definability of $\equiv_{c}^{\text {Ls }}$ (over every $c$, or only over $c=\varnothing$ ) is equivalent to thickness, which is discussed further in [Bena]. In Example $4.3, \equiv^{\text {Ls }}$ (over $\varnothing$ ) is not type-definable.

Definition 1.46. Let $a$ and $b$ be two hyperimaginaries.

We say that $a$ is definable over $b$ if $a$ is fixed by every automorphism of the universal domain fixing $b . \quad a$ is bounded over $b$ it is has boundedly many conjugates under automorphisms fixing $b$.

$\operatorname{bdd}(b)$ is (an arbitrary enumeration of) the set of all small hyperimaginaries (that is, quotients of tuples not longer than $|T|$ ) which are bounded over $b$. One easily sees that every hyperimaginary is interdefinable with a set of small hyperimaginaries, so a hyperimaginary is bounded over $b$ if and only if it is definable over bdd $(b)$.

Corollary 1.47. Let $\operatorname{tp}(a / c)$ be extendible. Then $a \equiv_{c}^{\mathrm{Ls}} b$ if and only if $a \equiv_{\operatorname{bdd}(c)} b$. In other words, an extendible Lascar strong type over $c$ is the same as an extendible type over $\operatorname{bdd}(c)$.

Proof. Having the same type over bdd $(b)$ is a bounded equivalence relation over $b$, whence one direction. Conversely, be Corollary 1.44, the set of pairs $\left(b, c^{\prime}\right)$ such that $c^{\prime}=c$ and $b \equiv_{c}^{\mathrm{Ls}} a$ is a hyperimaginary, and is clearly bounded over $c$ as such. The type of $a$ over this hyperimaginary clearly implies $\operatorname{lstp}(a / c)$.

$\mathrm{QED}_{1.47}$ 
Theorem 1.48 (Full independence theorem). Assume that $a_{0} \downarrow_{c} a_{1}, b_{i} \downarrow_{c} a_{i}$ for $i<$ $2, b_{0} \equiv_{c}^{\mathrm{Ls}} b_{1}$ and $\operatorname{tp}\left(b_{0} / c\right)$ is extendible. Then there is $b \downarrow_{c} a_{0} a_{1}$ with $b \equiv_{a_{i} c}^{\mathrm{Ls}} b_{i}$. Notice that we do not require $\operatorname{tp}\left(a_{i} / c\right)$ to be extendible.

Proof. We may assume that $\left\{a_{0}, a_{1}, b_{0}, b_{1}\right\}$ is a $c$-independent set, as we only need the extendibility of $\operatorname{tp}\left(b_{i} / c\right)$ for this. As $b_{0} \equiv_{c}^{\mathrm{Ls}} b_{1}$, they lie on a Morley sequence over c. As in the weak case, we apply Lemma 1.41 to replace $a_{0}$ by $a_{0}^{\prime} \downarrow_{c} a_{1} b_{1}$ such that $a_{0}^{\prime} b_{1} \equiv_{c} a_{0} b_{0}$ and $a_{0}^{\prime} \equiv_{a_{1 c}} a_{0}$. Send $a_{0}^{\prime}$ back to $a_{0}$ by an $a_{1} c$-automorphism, and let $b$ be the image of $b_{1}$. Then $b \downarrow_{c} a_{0} a_{1}$ and $b \equiv_{a_{i} c} b_{i}$ for $i<2$. In order to get $b \equiv_{a_{i} c}^{\mathrm{Ls}} b_{i}$, choose $b_{i}^{\prime}, b_{i}^{\prime \prime} \equiv_{a_{i} c}^{\mathrm{Ls}} b_{i}$ such that $\left\{a_{0}, a_{1}, b_{0}^{\prime}, b_{0}^{\prime \prime}, b_{1}^{\prime}, b_{1}^{\prime \prime}\right\}$ is $c$-independent. Then we have $a_{0} b_{0}^{\prime \prime} \downarrow_{c} a_{1} b_{1}^{\prime \prime}, b_{i}^{\prime} \downarrow_{c} a_{i} b_{i}^{\prime \prime}$ and $b_{0}^{\prime} \equiv_{c}^{\mathrm{Ls}} b_{1}^{\prime}$, so there is $b \downarrow_{c} a_{0} a_{1} b_{0}^{\prime \prime} b_{1}^{\prime \prime}$ with $b \equiv_{a_{i} b_{i}^{\prime \prime} c} b_{i}^{\prime}$. As $b_{i}^{\prime} \equiv{ }_{a_{i} c}^{\mathrm{Ls}} b_{i}^{\prime \prime}$, we get: $b \equiv_{a_{i} c}^{\mathrm{Ls}} b_{i}^{\prime \prime} \equiv_{a_{i} c}^{\mathrm{Ls}} b_{i}$.

$\mathrm{QED}_{1.48}$

1.5. Characterising independence relations. We consider what a relation of independence over $\mathcal{A}$-sets would be:

Definition 1.49. Let $\mathcal{A}$ be a co-final class, and $\Gamma$ a class of triplets $(a, b, c)$, where $a, b, c$ are possibly infinite tuples and $c \in \mathcal{A}$. Write $a\rfloor_{c}^{\Gamma} b$ for $(a, b, c) \in \Gamma$. Then $\Gamma$ is called a $\mathcal{A}$-independence relation, if it satisfies the following:

Invariance: $\Gamma$ is invariant under automorphisms. If $(a, b, c) \in \Gamma$, and $c^{\prime}, a^{\prime} c^{\prime}$ and $b^{\prime} c^{\prime}$ are interdefinable with $c, a c$ and $b c$, respectively, then $\left(a^{\prime}, b^{\prime}, c^{\prime}\right) \in \Gamma$.

Existence: $a \downarrow_{c}^{\Gamma} c$, for all $c \in \mathcal{A}$ and $a$.

Finite character: $a \mathbb{ل}_{c}^{\Gamma} b$ if and only if $a^{\prime} \mathfrak{l}_{c}^{\Gamma} b^{\prime}$ for all finite $a^{\prime} \subseteq a, b^{\prime} \subseteq b$.

Symmetry: $a \mathfrak{l}_{c}^{\Gamma} b$ if and only if $b \mathfrak{l}_{c}^{\Gamma} a$.

Restricted transitivity: If $b \in \mathcal{A}$ and $b c \in \mathcal{A}$, then $a \downarrow_{b} c d$ if and only if $a \downarrow_{b} c$ and $a \downarrow_{b c} d$.

Local character: There exists $\lambda$ such that whenever $\operatorname{cf}(\mu)>\lambda+|b|$, and $\left(c_{i}\right.$ : $i<\mu)$ is an increasing sequence of $\mathcal{A}$-sets, there is some $j<\mu$ with: $b \mathfrak{l}_{c_{j}}^{\Gamma} c_{<\mu}$.

Extension: If $a \downarrow_{c}^{\Gamma} b$ and $b^{\prime} \supseteq b$, then there is $a^{\prime} \equiv_{c} a$ with $a^{\prime} \mathfrak{l}_{c}^{\Gamma} b^{\prime}$.

Independence theorem: Whenever $c \in \mathcal{A}, a_{0} \mathbb{I}_{c}^{\Gamma} a_{1}, b_{i} \mathfrak{\Downarrow}_{c}^{\Gamma} a_{i}$ for $i<2$ and $b_{0} \equiv_{c}^{\mathrm{Ls}} b_{1}$, then there exists $b \downarrow_{c}^{\Gamma} a_{0} a_{1}$ with $b \equiv_{c a_{i}}^{\mathrm{Ls}} b_{i}$.

Remark 1.50. As we restrict to independence over $\mathcal{A}$-sets, we need to re-write the local character in the spirit of Proposition 1.17. Similarly, we need to restrict transitivity somewhat. In any case, our intention is to show that these conditions characterise simplicity and non-dividing, so the weaker the conditions the stronger the result, and once we proved this implies simplicity we have the full versions of transitivity and the local character at our disposal.

We prove (compare with [KP97]):

Theorem 1.51. A cat $T$ is simple if and only if there is an $\mathcal{A}$-independence relation $\Gamma$ for some co-final class $\mathcal{A}$. In this case, $T$ is $\mathcal{A}$-simple, and $\Gamma$ coincides with nondividing.

Proof. Left to right is known: in particular, $\mathcal{A}$ can be taken to be the class of all $\lambda$-compact models where $\lambda$ is given by Proposition 1.35.

We prove right to left: 
Claim. Assume that $c \in \mathcal{A}$ and $a\rfloor_{c}^{\Gamma} b$. Then $\operatorname{tp}(a / b c)$ does not divide over $c$.

Proof of claim. Let $\left(b_{i}\right)$ be a $c$-indiscernible sequence containing $b$. Take it to be very long, and for every $i$ find a $\mathcal{A}$-set $c_{i} \supseteq c \cup c_{<i} \cup\left\{b_{i}\right\}$ such that $b_{>i}$ is $c_{i}$-indiscernible. Assume that the sequence is of length $\kappa+1$, with $b=b_{\kappa}$. Taking $\kappa$ big enough, the local character gives us $i<\kappa$ such that $b \mathfrak{I}_{c_{i}}^{\Gamma} b_{<\kappa}$, which means that $\left(b_{j}: i<j<\kappa\right)$ is a $\Gamma$-Morley sequence over $c_{i}$ (a $\Gamma$-Morley sequence being the obvious thing). By extension, we may assume that $a \mathbb{ل}_{c}^{\Gamma} b c_{i}$, thus $a \mathbb{ل}_{c_{i}}^{\Gamma} b$ by restricted transitivity. Write $p^{\prime}(x, y)=\operatorname{tp}\left(a, b / c_{i}\right), p(x, y)=\operatorname{tp}(a, b / c)$. Repeated applications of the independence theorem show that we may find $a^{\prime}$ such that $\vDash \bigwedge_{i<j<i+\omega} p^{\prime}\left(a^{\prime}, b_{j}\right)$, so in particular $\vDash \bigwedge_{i<j<i+\omega} p\left(a^{\prime}, b_{j}\right)$.

$\mathrm{QED}_{\text {Claim }}$

Claim. $T$ is simple.

Proof of claim. Apply the local character of $\Gamma$, the previous claim and Proposition 1.17.

QED $_{\text {Claim }}$

Claim. For $c \in \mathcal{A}: a\rfloor_{c}^{\Gamma} b \Longleftrightarrow a \downarrow_{c} b$.

Proof of claim. Left to right was proved above.

For right to left, we can apply extension and the finite character to obtain a $\Gamma$-Morley sequence $\left(b_{i}: i<\kappa\right)$ over $c$ containing $b$. Since $a \downarrow_{c} b$, we may assume that $\left(b_{i}\right)$ is in fact $a c$-indiscernible. We will now construct a increasing sequence of $\mathcal{A}$-sets $\left(c_{i} \in \mathcal{A}: i<\kappa\right)$, beginning with $c_{0}=c$, such that $b_{i} \subseteq c_{i+1}$ and $b_{j} \mathbb{I}_{c} c_{i} b_{<j}$ for all $i \leq j<\kappa$.

For $i=0, c_{0}=c$ will do, since $b_{j} \downarrow^{\Gamma} b_{<j}$ by assumption. For $i>0$, let $c_{i}$ be an $\mathcal{A}$-set containing $c_{<i} b_{<i}$ such that $c_{i} \mathbb{N}_{c_{<i} b_{<i}} b_{<\kappa}$. By the induction hypothesis for $i$ we know that $b_{j} \mathbb{L}_{c} c_{<i} b_{<j}$ for all $i \leq j<\kappa$ (if $i$ is limit, use the finite character), so $b_{j} \mathbb{L}_{c} c_{i} b_{<j}$ as required.

By the local character applied to $\operatorname{tp}\left(a / \bigcup c_{i}\right)$, and assuming (as we may) that $\kappa$ was taken to be big enough, there is $i<\kappa$ such that:

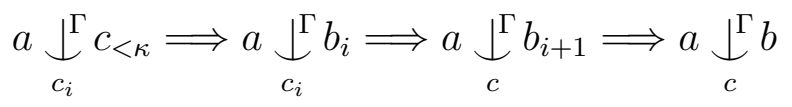

Which concludes the proof.

QED $_{\text {Claim }}$

Now the extension axiom for $\Gamma$ shows that $T$ is in fact $\mathcal{A}$-simple. $\quad$ QED $_{1.51}$

\section{Stability}

Here we sketch how the very basics of stability theory can be developed for cats. We do not pretend to give a full treatment, as a much more complete one already exists in [She75]: our humble goal is to show the connection with simplicity, and we only develop those tools needed for it.

Definition 2.1. $\quad$ (i) Let $\varphi(x, y), \psi(x, y)$ be two formulas. We define the local $R(-, \varphi, \psi, \lambda)$ rank, by induction. The interesting part is: $R(p, \varphi, \psi, \lambda) \geq \alpha+1$ if there are $\left(p_{i}: i<\lambda\right)$ extending $p$, with $R\left(p_{i}, \varphi, \psi, \lambda\right) \geq \alpha$ for all $i<\lambda$, and such the for all $i<j<\lambda$ there is $b$ such that $\varphi(x, b) \in p_{i}, \psi(x, b) \in p_{j}$ 
or the other way around, and $\varphi$ and $\psi$ are contradictory (so if they are not contradictory, $R(p, \varphi, \psi, \lambda)$ is either 0 or -1$)$.

(ii) The pair $\varphi, \psi$ is stable if $R(x=x, \varphi, \psi, 2)<\omega$. This is equivalent to: $R(x=$ $x, \varphi, \psi, \omega)<\omega$.

(iii) The formula $\varphi$ is stable if $\varphi, \psi$ are, for all $\psi$ on the same tuple of variables.

(iv) $T$ is stable if all formulas are, that is if all pairs are.

(v) A $\varphi(x, y)$-definition for $p(x) \in \mathrm{S}(A)$ is a partial existential type $q(y)$ over $A$, containing at most $|T|$ formulas, such that for all $b \in A: p \vdash \varphi(x, b)$ if and only if $q(b)$. It is sometimes noted $d_{p} \varphi$. A type is $\varphi$-definable if it has a $\varphi$-definition.

(vi) A definition for $p$ is a set $\left\{d_{p} \varphi\right\}$ such that $d_{p} \varphi$ is a $\varphi$-definition for $p$, for all relevant $\varphi$. A type is definable if it has a definition.

(vii) A definition $\left\{d_{p} \varphi\right\}$ for $p$ is good if when applied to any set $B$ it gives a complete consistent type $p^{\prime} \in \mathrm{S}(B)$. A type is well definable if it has a good definition.

Proposition 2.2. The following are equivalent:

(i) $\varphi$ is stable.

(ii) Any complete type has a $\varphi$-definition.

(iii) For all $A$ there are at most $(|A|+|T|)^{|T|}$ different (positive) $\varphi$-types in $\mathrm{S}(A)$.

(iv) For some $\lambda$, for all $A$ such that $|A| \leq \lambda$, there are at most $\lambda$ different (positive) $\varphi$-types in $\mathrm{S}(A)$.

Proof. $\quad($ i $) \Longrightarrow$ (ii). Let $\psi$ be any formula on the right tuple that contradicts $\varphi$, and let $n=R(p, \varphi, \psi, 2)$. Considering trees, compactness yields that there is some $\chi \in p$ with $n=R(\chi, \varphi, \psi, 2)$. Then $R(\chi \wedge \varphi(x, y), \varphi, \psi, 2) \geq n$ can be written as an existential formula in $y$. Let $d_{p} \varphi$ be the conjunction of all formulas arising this way, and we claim that it has the required properties. Indeed, suppose that $\varphi(x, b) \in p$. Then we have $\chi \wedge \varphi(x, b) \in p$ as well, so it has rank $n$. On the other hand, if $\varphi(x, b) \notin p$, there must be some $\psi(x, b) \in p$ with $\psi$ contradicting $\varphi$. Then we get $R(\chi \wedge \psi(x, b), \varphi, \psi, 2)=n$, so necessarily $R(\chi \wedge \varphi(x, b), \varphi, \psi, 2)<n$, as otherwise $R(\chi, \varphi, \psi, 2)>n$. Since there are $|T|$ possible choices for $\psi,\left|d_{p} \varphi\right| \leq|T|$ as required.

(ii) $\Longrightarrow$ (iii). Over a set $A$ there are at most $(|A|+|T|)^{|T|}$ possible definitions, given the bound on the size of one.

(iii) $\Longrightarrow$ (iv). Clear.

(iv) $\Longrightarrow$ (i). The standard proof: suppose that $R(x=x, \varphi, \psi, 2) \geq \omega$ for some $\psi$. For any $\lambda$, let $\mu$ be minimal such that $2^{\mu}>\lambda$. Build a tree of depth $\mu$ : it has $\leq \lambda$ parameters, and $2^{\mu}>\lambda$ branches, that is partial types that when completed necessarily give different $\varphi$-types.

$\mathrm{QED}_{2.2}$

We get then the usual type counting characterisation for stable theories.

We now point out the connection between stability and simplicity, using several ideas from [Pil98].

Lemma 2.3. ( $T$ simple)

An extendible stationary type is well definable. In fact, it has a definition that gives its unique non-dividing extension to any set containing its domain. 
Proof. Let $p=\operatorname{tp}(a / C)$ be a stationary and extendible. For each $\varphi(x, y)$, let $d_{p}^{\prime} \varphi(y)$ be the existential type that says that $D(p(x) \wedge \varphi(x, y), \varphi, \psi)=D(p, \varphi, \psi)$ whenever that makes sense. Then by the stationarity, the $d_{p}^{\prime} \varphi$ define on $B \supseteq C$ the unique non-dividing extension of $p$ to $B$, noted $p\lceil B$. Of course, there is no reason to assume that $\left|d_{p}^{\prime} \varphi\right| \leq|T|$, which is required for it to be a definition for $p$, so there is some more work to be done.

For all contradicting pairs $\varphi, \chi$, consider $d_{p}^{\prime} \varphi \wedge d_{p}^{\prime} \chi$ : Clearly, this must be inconsistent, otherwise some non-dividing extension of $p$ would satisfy $\varphi(x, b) \wedge \chi(x, b)$. By compactness (which applies to existential formulas as well), there are finitely many formulas in $d_{p}^{\prime} \varphi, d_{p}^{\prime} \chi$ that give this contradiction. Take $d_{p} \varphi$ to contain all formulas that arise this way in $d_{p}^{\prime} \varphi$, for all $\chi$. Then $\left|d_{p} \varphi\right| \leq|T|$ as required, and $\left\{d_{p} \varphi\right\}$ gives a consistent type over any $B \supseteq C$, that contains the unique non-dividing extension, and must then be equal to it. In particular, on $C$ it gives $p$.

$\mathrm{QED}_{2.3}$

Corollary 2.4. ( $T$ simple)

An extendible type of bounded multiplicity is definable. In fact, it has a definition that gives the intersection of its non-dividing extensions to any set containing its domain.

Proof. Let $p=\operatorname{tp}(a / C)$ be extendible of bounded multiplicity. Then it has a stationary non-dividing extension $q$ to some $B \supseteq C$. Let $\left\{d_{q} \varphi\right\}$ be the (good) definition of $q$ giving its non-dividing extensions. Then for any set $C^{\prime} \supseteq C$, applying this definition to $C^{\prime}$ gives a non-dividing extension of $p$. Let $b \subseteq B$ be the tuple of parameters occurring in this definition, so write it rather $\left\{d_{q} \varphi(y, b)\right\}$. Let $b^{\prime}$ be a $C$-conjugate of $b$. Then $\left\{d_{q} \varphi\left(y, b^{\prime}\right)\right\}$ also gives non-dividing extensions of $p$. Since $p$ has bounded multiplicity, there is a set $\bar{b}=\left\{b_{i}\right\}$ of $C$-conjugates of $b$ such that for any other $C$-conjugate $b^{\prime}$ of $b$, the definition $\left\{d_{q} \varphi\left(y, b^{\prime}\right)\right\}$ is already equivalent to $\left\{d_{q} \varphi\left(y, b_{i}\right)\right\}$ for some $i$. Write: $r(z)=\operatorname{tp}(b / C), \bar{r}(\bar{z})=\operatorname{tp}(\bar{b} / C)$, and:

$$
\begin{aligned}
& d_{p}^{\prime} \varphi(y)=\exists z r(z) \wedge d_{q} \varphi(y, z) \\
& d_{p}^{\prime \prime} \varphi(y)=\exists \bar{z} \bar{r}(\bar{z}) \wedge \bigwedge_{i} d_{q} \varphi\left(y, z_{i}\right)
\end{aligned}
$$

Clearly these can be written as existential types over $C$. So $d_{p}^{\prime} \varphi(y)$ says that there is some non-dividing extension of $p$ containing $\varphi(x, y)$, and $d_{p}^{\prime \prime} \varphi(y)$ says that all nondividing extensions of $p$ contain $\varphi(x, y)$. We therefore see that if $\varphi$ and $\chi$ are contradictory, then $d_{p}^{\prime} \varphi(y) \wedge d_{p}^{\prime \prime} \chi(y)$ is inconsistent. By compactness (applied to existential formulas), finitely many formulas suffice for the contradiction. Take $d_{p} \varphi$ to be the set of all formulas arising this way in $d_{p}^{\prime \prime} \varphi$. Then $\left|d_{p} \varphi\right| \leq|T|$ as required, and we claim that it is equivalent to $\left\{d_{p}^{\prime \prime} \varphi\right\}$. Indeed, suppose that $c \not \models d_{p}^{\prime \prime} \varphi$. Then there is some nondividing extension of $p$ to $C c$ that does not satisfy $\varphi(x, c)$, so it must satisfy $\chi(x, c)$ for some $\chi$ contradicting $\varphi$. Then we have $c \vDash d_{p}^{\prime} \chi$, so by construction: $c \not \models d_{p} \varphi$. This proves the claim. In particular, $d_{p} \varphi$ gives $p$ on $C$.

$\mathrm{QED}_{2.4}$

We recall that if $A \supseteq B$, and $p \in \mathrm{S}(A)$ is such that whenever $a, a^{\prime} \in A, a \equiv_{B} a^{\prime}$, then $p \vdash \varphi(x, a) \Longleftrightarrow p \vdash \varphi\left(x, a^{\prime}\right)$, then $p$ is called non-splitting over $B$. We usually consider this property only when $B$ is a model and $A$ satisfies at least some saturation requirements over $B$.

Notation 2.5. $\mathcal{M}_{c t}$ is the class of $|T|^{+}$-compact models of $T$. 
Lemma 2.6. Let $M \in \mathcal{M}_{c t}$, and suppose that $p \in \mathrm{S}(M)$ is definable. Then:

(i) $p$ has a unique definition over $M$ (up to equivalence).

(ii) The unique definition of $p$ is good.

(iii) For $A \supseteq M, p\lceil A$ (the interpretation of the definition of $p$ on $A$ ) does not divide over $M$, and is non-splitting over $M$.

Proof. (i) Suppose there are two inequivalent $\varphi(x, y)$-definitions $q(y)$ and $q^{\prime}(y)$ for $p$. Then there is some $a$ realising, say, $q$ and not $q^{\prime}$. Then there is a contradicting pair $\psi, \chi$ and $b \in M$ such that $\psi(y, b) \in q^{\prime}$, and $\vDash \chi(a, b)$. Then $q(y) \wedge \chi(y, b)$ is consistent, and by $|T|^{+}$-compactness it is realised in $M$, contradicting the assumption that $q, q^{\prime}$ were the same on $M$.

(ii) For $A \supseteq M$, let $p\lceil A$ denote the application of the (unique) definition of $p$ to $A$. We show first that $p\left\lceil A\right.$ is consistent. Indeed, assume that $\left\{\varphi_{i}\left(x, a_{i}\right)\right.$ : $i<n\} \subseteq p \nmid A$. This means that for $i<n: a_{i} \vDash d_{p} \varphi_{i}$. Let $B \subseteq M$ be the set of parameters used for $\left\{d_{p} \varphi_{i}: i<n\right\}$. Then $|B| \leq|T|$, so $\operatorname{tp}\left(a_{<n} / B\right)$ is realised in $M$, say by $b_{<n}$. Thus $p \vDash \bigwedge \varphi_{i}\left(x, b_{i}\right)$, and $\bigwedge \varphi_{i}\left(x, b_{i}\right)$ is consistent. As $a_{<n} \equiv b_{<n}$, so is $\bigwedge \varphi_{i}\left(x, a_{i}\right)$, so $p \nmid A$ is consistent.

To prove completeness, suppose that for some formulas $\varphi_{i}(x, y): i<\lambda \leq|T|$ we have $\vDash \bigvee_{i<\lambda} \varphi_{i}$, and let $B \subseteq M$ contain all the parameters for $d_{p} \varphi_{i}: i<\lambda$. Then $|B| \leq|T|$, so for every $a \in A$, there is $b \in M$ realising $\operatorname{tp}(a / B)$. Then, as $p$ is complete, for some $i<\lambda$ we have:

$$
p \vdash \varphi_{i}(x, b) \Longrightarrow b \vDash d_{p} \varphi_{i} \Longrightarrow a \vDash d_{p} \varphi_{i} \Longrightarrow p \uparrow A \vdash \varphi_{i}(x, a)
$$

which proves that $p\lceil A$ is complete.

(iii) Easy.

$\mathrm{QED}_{2.6}$

Corollary 2.7. (T stable)

The equality of Lascar strong types over $A$ is the transitive closure of equality of types over $\mathcal{M}_{c t}$-models containing $A$.

Proof. We have non-splitting extensions over $\mathcal{M}_{c t}$-models (see [KP97]).

$\mathrm{QED}_{2.7}$

In particular, if $T$ is stable, the Lascar strong type over $\mathcal{M}_{c t}$-models is the same as the usual type.

Theorem 2.8. The following are equivalent:

(i) $T$ is stable.

(ii) $T$ is $\mathcal{M}_{c t}$-simple, and $\mathcal{M}_{c t}$-types are stationary.

(iii) $T$ is simple, and extendible types have bounded multiplicity.

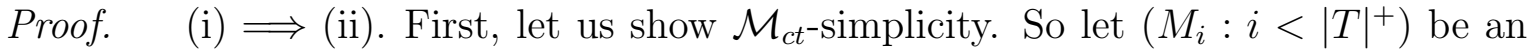
increasing sequence, and $p \in \mathrm{S}_{n}\left(M_{<|T|^{+}}\right)$. Consider a definition of $p$ : it contains at most $|T|$ parameters, therefore it is over some $M_{j}$, so it is a good definition and $p$ does not divide over $M_{j}$. This shows that $T$ is simple. The existence of Morley sequences results from the existence of non-splitting extensions.

Suppose now that there is some $\mathcal{M}_{c t}$-type $p \in \mathrm{S}(M)$ which is not stationary. That is, there is some $a$ and two different non-dividing extensions $p_{0}, p_{1} \in$ $\mathrm{S}(M a)$ of $p$. Let $\left(a_{i}: i<\lambda\right)$ be a Morley sequence in $\operatorname{tp}(a / M)$. Then by 
the independence theorem for Lascar strong types ( $p$ is one by Corollary 2.7), for every $\varepsilon \in 2^{\lambda}$ there is $b_{\varepsilon} \downarrow_{M} a_{<\lambda}$ such that $b_{\varepsilon} \vDash \bigcup_{i<\lambda} p_{\varepsilon_{i}}\left(x, a_{i}\right)$. Thus, disposing of $M$ (except maybe for a finite set of elements needed to establish the difference between $p_{0}$ and $p_{1}$ ), we obtain $2^{\lambda}$ types over sets of cardinality $\lambda$, for any $\lambda$, contradicting stability.

(ii) $\Longrightarrow$ (iii). Clear, when considering the non-dividing extensions of an extendible type to an $\mathcal{M}_{c t}$-model containing its domain.

(iii) $\Longrightarrow$ (i). Since extendible types have bounded multiplicity, we can find for every set $B$ a superset $B^{\prime} \supseteq B$ such that every extendible type over $B^{\prime}$ non-dividing over $B$ is stationary. By Proposition 1.35, there is some $\lambda$ such that every type over a $\lambda$-compact model is extendible, and we may assume that $\lambda \geq|T|^{+}$is big enough so that if $|B| \leq|T|$ then there is $B^{\prime}$ as above with $\left|B^{\prime}\right|<\lambda$.

We claim that $T$ is $\mu$-stable for every $\mu$ satisfying $\mu^{<\lambda}=\mu$ (so for example, for $\mu=2^{\lambda}$ ).

Assume that $|A|=\mu=\mu^{<\lambda}$ : then there is a $\lambda$-compact model $M$ containing $A$ of cardinality $\mu$, and it would suffice to show that $\left|\mathrm{S}_{n}(M)\right| \leq \mu$. Every $p \in \mathrm{S}_{n}(M)$ is extendible, and does not divide over some $B \subseteq M,|B| \leq|T|$, which gives $\mu^{|T|}=\mu$ possibilities. By assumption on $\lambda$, we can choose every such $B$ a superset $B \subseteq B^{\prime} \subseteq M$ as above. Then $p \uparrow B^{\prime}$ is stationary as it is extendible and does not divide over $B$, and $p$ is its unique non-dividing extension to $M$. This leaves us still with $\left|\mathrm{S}_{n}\left(B^{\prime}\right)\right| \leq 2^{<\lambda} \leq \mu$ possibilities, and the statement is proved.

$\mathrm{QED}_{2.8}$

\section{Canonical bases}

When trying to generalise the construction of canonical bases from [HKP00] to cats, one encounters several technical difficulties:

- The proof that the relation $\sim_{1}$ (having a common non-dividing extension) is type-definable does not go through: if $p(x, a)$ and $p^{\prime}\left(x, a^{\prime}\right)$ are two complete types, and $D\left(p \wedge p^{\prime}, \varphi, \psi\right)=D(p, \varphi, \psi)=D\left(p^{\prime}, \varphi, \psi\right)$ for all $\varphi, \psi$, then we still do not know whether $p \sim_{1} p^{\prime}$, if neither $\operatorname{tp}\left(a / a^{\prime}\right)$ nor $\operatorname{tp}\left(a^{\prime} / a\right)$ is extendible.

- The definition of the relation $R$ uses negative formulas (namely, the negations of formulas that make $D$-ranks decrease) which we do not allow. Therefore, with the definitions of [HKP00], it is not even clear that $R$ is consistent.

- The proof in [HKP00] makes use of instances of the extension axiom which may no longer be valid in our context.

We suggest therefore an alternative approach, similar in spirit but different in details: the relation $R$ is defined quite differently (although it turns out to be the same), and we do not even claim that it is type-definable; the type-definability of $\sim_{1}$ is proved differently; and applications of the extension axiom are replaced with more direct arguments. This is accomplished through an extensive use of Morley sequences in the types we consider, and of Proposition 1.32.

In order to apply this approach, all we need is a simple cat $T$ and an extendible amalgamation base $p$. In particular, $p$ does not divide over $\mathrm{Cb}(p)$, so $p \uparrow \mathrm{Cb}(p)$ is extendible and independence theory applies to it in full. 
Convention 3.1. $\quad$ (i) $T$ is simple.

(ii) In this section a complete type over $a$ is noted $p(x, a)$, where $p(x, y)$ is the obvious complete pure type.

Definition 3.2. (i) An extendible type $p(x, a)$ is an amalgamation base if it satisfies the independence theorem. Equivalently: if it has a unique extension to $\operatorname{bdd}(a)$.

(ii) Let $p(x, a)$ and $p^{\prime}\left(x, a^{\prime}\right)$ be amalgamation base, and $\left(c_{i}: i<\omega\right)$ a Morley sequence in both (over $a$ and over $a^{\prime}$, respectively), indiscernible over $a a^{\prime}$. Then we say that $c_{<\omega}$ witnesses that $p(x, a) \sim_{1} p^{\prime}\left(x, a^{\prime}\right)$.

If in addition $a \downarrow_{c_{<\omega}} a^{\prime}$, then $c_{<\omega}$ witnesses that $p(x, a) R p^{\prime}\left(x, a^{\prime}\right)$.

(iii) We define $\sim$ as the transitive closure of $\sim_{1}$, and we say that $p \sim p^{\prime}$ are parallel.

The first step is to show that the relation $p(x, a) \sim_{1} p^{\prime}\left(x, a^{\prime}\right)$ is type-definable (for a given pair of $p(x, y)$ and $\left.p^{\prime}\left(x, y^{\prime}\right)\right)$.

Lemma 3.3. Let $p(x, a)$ and $p^{\prime}\left(x, a^{\prime}\right)$ be amalgamation bases, and $q\left(x_{<\omega}, a\right)$ the type of a Morley sequence in $p$. Then the following are equivalent:

(i) $p \sim_{1} p^{\prime}$.

(ii) $p$ and $p^{\prime}$ have a common non-dividing extension to $a a^{\prime}$.

(iii) $D(p, \varphi, \psi)=D\left(p^{\prime}, \varphi, \psi\right)$ for every $\varphi, \psi$, and $q\left(x_{<\omega}, a\right) \wedge \bigwedge_{i<\omega} p^{\prime}\left(x^{\prime}, a^{\prime}\right)$ is consistent.

Proof. (i) $\Longrightarrow$ (ii). If $c_{<\omega}$ witnesses that $p \sim_{1} p^{\prime}$, then $\operatorname{tp}\left(c_{0} / a a^{\prime}\right)$ is a common nondividing extension by Proposition 1.32 .

(ii) $\Longrightarrow$ (iii). Let $c$ realise a common non-dividing extension, namely $c \vDash p \wedge p^{\prime}$ and $c \downarrow_{a} a^{\prime} \wedge c \downarrow_{a^{\prime}} a$. Then $D(p, \varphi, \psi)=D(c / a, \varphi, \psi)=D\left(c / a a^{\prime}, \varphi, \psi\right)=$ $D\left(c / a^{\prime}, \varphi, \psi\right)=D\left(p^{\prime}, \varphi, \psi\right)$. As $\operatorname{tp}\left(a^{\prime} / a c\right)$ does not divide over $a, q\left(x_{<\omega}, a\right) \wedge$ $\bigwedge_{i<\omega} p^{\prime}\left(x^{\prime}, a^{\prime}\right)$ is consistent.

(iii) $\Longrightarrow$ (i). Assume that $q\left(x_{<\omega}, a\right) \wedge \bigwedge_{i<\omega} p^{\prime}\left(x^{\prime}, a^{\prime}\right)$ is consistent. By compactness and Lemma 1.2, we can find an $a a^{\prime}$-indiscernible sequence $c_{<\omega}$ realising it. By Proposition $1.32 c_{<\omega} \downarrow_{a} a^{\prime}$, so $c_{i} \downarrow_{a} a^{\prime} c_{<i}$ for every $i<\omega$, and whereby for every $\varphi, \psi$ :

$$
\begin{aligned}
D\left(c_{i} / a^{\prime} c_{<i}, \varphi, \psi\right) & \geq D\left(c_{i} / a a^{\prime} c_{<i}, \varphi, \psi\right)=D\left(c_{i} / a, \varphi, \psi\right)=D(p, \varphi, \psi) \\
& =D\left(p^{\prime}, \varphi, \psi\right)=D\left(c_{i} / a^{\prime}, \varphi, \psi\right)
\end{aligned}
$$

We conclude that $c_{i} \downarrow_{a^{\prime}} c_{<i}$, so $c_{<\omega}$ is a common Morley sequence witnessing $p \sim_{1} p^{\prime}$.

$\mathrm{QED}_{3.3}$

Corollary 3.4. Let $p(x, a)$ and $p^{\prime}\left(x, a^{\prime}\right)$ be amalgamation bases. Then the relation $p(x, b) \sim_{1} p^{\prime}\left(x, b^{\prime}\right)$ is type-definable in $b, b^{\prime}$.

Proof. If there are $\varphi, \psi$ such that $D(p(x, a), \varphi, \psi) \neq D\left(p^{\prime}\left(x, a^{\prime}\right), \varphi, \psi\right)$, then the relation is empty. Otherwise, if $q(x, a)$ is the type of any Morley sequence in $p(x, a)$, then $p(x, b) \sim_{1} p^{\prime}\left(x, b^{\prime}\right) \Longleftrightarrow \vDash \exists x_{<\omega} q\left(x_{<\omega}, b\right) \wedge \bigwedge_{i<\omega} p^{\prime}\left(x^{\prime}, b^{\prime}\right)$.

$\mathrm{QED}_{3.4}$

Notation 3.5. Let $c_{<\alpha+1}$ be an $a$-indiscernible sequence, $\alpha \geq \omega$. Then we note $\operatorname{tp}_{\text {next }}\left(c_{<\alpha}\right)=\operatorname{tp}\left(c_{\alpha} / c_{<\alpha}\right)$, that is the type of the next element of the sequence. As $\alpha \geq \omega$, this does not depend on the choice of $c_{\alpha}$. 
The proof of the main result is by induction. The starting point is given by:

Lemma 3.6. Define $\alpha^{*}=\left\{\beta^{*}: \beta \in \alpha\right\}$ for every ordinal $\alpha$ (by induction on $\alpha$ ), and define that $\alpha^{*}<\beta^{*} \Longleftrightarrow \beta<\alpha$ and $\alpha<\beta^{*}$ for every two ordinals $\alpha, \beta$.

If $\left(c_{i}: i \in \omega \cup \omega^{*}\right)$ is an indiscernible sequence, then $\operatorname{tp}_{\text {next }}\left(c_{\in \omega}\right)$ is an amalgamation base, and $c_{\in \omega^{*}}$ is a Morley sequence in it (over $c_{\in \omega}$ ).

Proof. For $i^{*} \in \omega^{*}, \operatorname{tp}\left(c_{i^{*}} / c_{\in \omega} c_{\in i^{*}}\right)$ does not divide over $c_{\in \omega}$, as every formula it contains is already satisfied by some element of $c_{\in \omega}$. This shows that $c_{\in \omega^{*}}$ is Morley sequence in $\operatorname{tp}_{\text {next }}\left(c_{\in \omega}\right)$, and in particular $\operatorname{tp}_{\text {next }}\left(c_{\in \omega}\right)$ is extendible. If $c, c^{\prime} \vDash \operatorname{tp}_{\text {next }}\left(c_{\in \omega}\right)$, then we can extend $c_{\in \omega}$ to $c_{\in 2 \omega}$ and $c_{\in 2 \omega}^{\prime}$, such that $c_{\in \omega+1}^{\prime}=c_{\in \omega+1}, c=c_{\omega+1}$ and $c^{\prime}=c_{\omega+1}^{\prime}$, whereby $c \equiv_{c \in \omega}^{\mathrm{Ls}} c^{\prime \prime} \equiv_{c \in \omega}^{\mathrm{Ls}} c^{\prime}$, so tp $p_{\text {next }}\left(c_{\in \omega}\right)$ is an amalgamation base. $\quad \mathrm{QED}_{3.6}$

Corollary 3.7. Let $p(x, a)$ be an amalgamation base, and $c_{<\omega}$ a Morley sequence in $p$. Then $p R \mathrm{tp}_{\text {next }}\left(c_{<\omega}\right)$.

Proof. We may extend it to a sequence $\left(c_{i}: i \in \omega \cup \omega^{*}\right)$, and apply the lemma. Then $\operatorname{tp}_{\text {next }}\left(c_{<\omega}\right)$ is an amalgamation base, and $c_{\in \omega^{*}}$ is a Morley sequence in $p$ and in $\operatorname{tp}_{\text {next }}\left(c_{<\omega}\right)$, indiscernible over $a c_{<\omega}$. But by symmetry of $\omega \cup \omega^{*}, c_{<\omega}$ is a Morley sequence over $c_{\in \omega^{*}}$, indiscernible over $a c_{\in \omega^{*}}$, so $c_{<\omega} \downarrow_{c_{\in \omega^{*}}} a$. We conclude that $c_{\in \omega^{*}}$ witnesses that $p R$ tp $_{\text {next }}\left(c_{<\omega}\right)$.

$\mathrm{QED}_{3.7}$

And the induction step is given by:

Lemma 3.8. Assume that $a \downarrow_{a^{\prime}} a^{\prime \prime}$ and $p(x, a) R p^{\prime}\left(x, a^{\prime}\right) \sim_{1} p^{\prime \prime}\left(x, a^{\prime \prime}\right)$. Then $a \downarrow_{a^{\prime \prime}} a^{\prime}$ and $p(x, a) R p^{\prime \prime}\left(x, a^{\prime \prime}\right)$.

Proof. Let $c_{<\omega}$ witness $p R p^{\prime}$. We may extend it to an $a a^{\prime}$-indiscernible sequence $c_{<\lambda}$ of any length $\lambda$. Let $q\left(x, a^{\prime} a^{\prime \prime}\right)$ be a common non-dividing extension of $p^{\prime}$ and $p^{\prime \prime}$.

By induction on $i<\lambda$, we choose a sequence $\left(d_{i}: i<\lambda\right)$ such that:

(i) $d_{i} \vDash q$ for every $i<\lambda$.

(ii) $d_{<\lambda} \equiv_{a a^{\prime}} c_{<\lambda}$.

(iii) $d_{<\lambda} \downarrow_{a^{\prime}} a a^{\prime \prime}$

Assume we have already chosen $d_{<i} \equiv_{a a^{\prime}} \quad c_{<i}$ such that $d_{<i} \downarrow_{a^{\prime}} a a^{\prime \prime}$. Write $q^{\prime}\left(x, a a^{\prime} c_{<i}\right)=\operatorname{tp}\left(c_{i} / a a^{\prime} c_{<i}\right)$. Then both $q^{\prime}\left(x, a a^{\prime} d_{<i}\right)$ and $q\left(x, a^{\prime} a^{\prime \prime}\right)$ are non-dividing extensions of $p^{\prime}$, and $a \downarrow_{a^{\prime}} a^{\prime \prime} \Longrightarrow a d_{<i} \downarrow_{a^{\prime}} a^{\prime \prime}$. Since $p^{\prime}$ is an amalgamation base we can find $d_{i} \downarrow_{a^{\prime}} a a^{\prime \prime} d_{<i}$ realising $q^{\prime}\left(x, a a^{\prime} d_{<i}\right) \wedge q\left(x, a^{\prime} a^{\prime \prime}\right)$, and the construction may continue.

Having chosen $\lambda$ big enough, we may assume by Lemma 1.2 that the sequence $d_{<\omega}$ is $a a^{\prime} a^{\prime \prime}$-indiscernible, in which case it is a Morley sequence in all of $p, p^{\prime}$ and $p^{\prime \prime}$, and is independent of $a a^{\prime} a^{\prime \prime}$ over either one of $a, a^{\prime}$ or $a^{\prime \prime}$. Therefore:

$$
d_{<\omega} \underset{a^{\prime}}{\downarrow} a a^{\prime \prime} \Longrightarrow a d_{<\omega} \underset{a^{\prime}}{\downarrow} a^{\prime \prime} \Longrightarrow a \underset{d<\omega}{\downarrow} a^{\prime} a^{\prime \prime} \Longrightarrow a d_{<\omega} \underset{a^{\prime \prime}}{\downarrow} a^{\prime}
$$

Thus the sequence $d_{<\omega}$ witnesses that $p(x, a) R p^{\prime \prime}\left(x, a^{\prime \prime}\right)$, and $a \downarrow_{a^{\prime \prime}} a^{\prime}$.

$\mathrm{QED}_{3.8}$

We put them together:

Lemma 3.9. Assume that $p(x, a) \sim p^{\prime}\left(x, a^{\prime}\right)$, and $c_{<\omega}$ is a Morley sequence in $p$ such that $c_{<\omega} \downarrow_{a} a^{\prime}$. Then $p(x, a) R \operatorname{tp}_{\text {next }}\left(c_{<\omega}\right) R p^{\prime}\left(x, a^{\prime}\right)$ and $c_{<\omega} \downarrow_{a^{\prime}} a$. 
Proof. We have $p(x, a)=p_{0}\left(x, a_{0}\right) \sim_{1} \ldots \sim_{1} p_{n}\left(x, a_{n}\right)=p^{\prime}\left(x, a^{\prime}\right)$, and as $c_{<\omega} \downarrow_{a} a^{\prime}$, we may further assume that $c_{<\omega} \downarrow_{a} a_{\leq n}$.

Then $\operatorname{tp}_{\text {next }}\left(c_{<\omega}\right) R p(x, a)$ by Corollary 3 , and we may apply Lemma 3.8 repeatedly to obtain $\operatorname{tp}_{\text {next }}\left(c_{<\omega}\right) R p_{i}$ and $c_{<\omega} \downarrow_{a_{i}} a_{\leq n}$ for every $i \leq n$. For $i=n$, this is what we want.

$\mathrm{QED}_{3.9}$

Proposition 3.10. Let $p(x, a)$, and $p^{\prime}\left(x, a^{\prime}\right)$ be amalgamation bases. Let $c_{<\omega}$ be $a$ Morley sequence in $p$, and $p^{\prime \prime}\left(x, c_{<\omega}\right)=\operatorname{tp}_{\text {next }}\left(c_{<\omega}\right)$. Then the following are equivalent:

(i) $p(x, a) \sim p^{\prime}\left(x, a^{\prime}\right)$

(ii) There exists a sequence $d_{<\omega}$ such that $p(x, a) R p^{\prime \prime}\left(x, d_{<\omega}\right) R p^{\prime}\left(x, a^{\prime}\right)$, and in addition $d_{<\omega} \downarrow_{a^{\prime}}$ a and $d_{<\omega} \downarrow_{a} a^{\prime}$.

(iii) There exists a sequence $d_{<\omega}$ such that $p(x, a) \sim_{1} p^{\prime \prime}\left(x, d_{<\omega}\right) \sim_{1} p^{\prime}\left(x, a^{\prime}\right)$.

Proof. $\quad$ (i) $\Longrightarrow$ (ii). We may choose $d_{<\omega} \equiv_{a} c_{<\omega}$ such that $d_{<\omega} \downarrow_{a} a^{\prime}$, and apply Lemma 3.9.

(ii) $\Longrightarrow$ (iii). Clear, as $R$ implies $\sim_{1}$.

(iii) $\Longrightarrow$ (i). By definition.

$\mathrm{QED}_{3.10}$

Corollary 3.11. Let $p(x, a)$ be an amalgamation base, and $q=\operatorname{tp}(a)$. For $a^{\prime}, a^{\prime \prime} \vDash q$ define $a^{\prime} \sim a^{\prime \prime}$ if $a^{\prime}=a^{\prime \prime}$ or $p\left(x, a^{\prime}\right) \sim p\left(x, a^{\prime \prime}\right)$. Then $\sim$ is a type-definable equivalence relation.

Proof. Clearly, $\sim$ is an equivalence relation. Let $c_{<\omega}$ be a Morley sequence in $p(x, a)$, and $p^{\prime}\left(x, c_{<\omega}\right)=\operatorname{tp}_{\text {next }}\left(c_{<\omega}\right)$. Then $a^{\prime} \sim a^{\prime \prime}$ if and only if $a^{\prime}=a^{\prime \prime}$ or there is a sequence $d_{<\omega}$ such that $p\left(x, a^{\prime}\right) \sim_{1} p^{\prime}\left(x, d_{<\omega}\right) \sim_{1} p\left(x, a^{\prime \prime}\right)$, and this is type-definable. $\mathrm{QED}_{3.11}$

Therefore, we can give:

Definition 3.12. For an extendible amalgamation base $p(x, a)$ we define the canonical base of $p, \mathrm{Cb}(p)$, as the hyperimaginary $a_{\sim}$.

Note that in the beginning of the proof of the following theorem we have to turn around a bit, as we do not know that $\operatorname{tp}\left(a / a_{\sim}\right)$ is extendible.

Theorem 3.13. Let $p(x, a)$ be an extendible amalgamation base, $a_{\sim}=\mathrm{Cb}(p)$. Then:

(i) For $a^{\prime} \equiv a$, we have $p(x, a) \sim p\left(x, a^{\prime}\right)$ if and only if $\mathrm{Cb}(p(x, a))=\mathrm{Cb}\left(p\left(x, a^{\prime}\right)\right)$. Thus $\operatorname{Cb}(p)$ is a canonical parameter for the parallelism class of $p$.

(ii) $p$ does not divide over $a_{\sim}$. Equivalently, if $c_{<\omega}$ is a Morley sequence in $p$, then $c_{<\omega} \downarrow_{a} a$.

(iii) $p(x, a) \stackrel{\sim}{R} p^{\prime}\left(x, a^{\prime}\right)$ if and only if $p \sim p^{\prime}$ and $a \downarrow_{a \sim} a^{\prime}$.

(iv) $p\lceil\mathrm{Cb}(p)$ is an amalgamation base.

(v) If $b \subseteq a$, then $p$ does not divide over $b$ if and only if $a_{\sim} \subseteq \operatorname{bdd}(b)$. If in addition $p\left\lceil b\right.$ is an amalgamation base, then $a_{\sim} \subseteq b$.

Proof. $\quad$ (i) By definition.

(ii) Let $c_{<\omega}$ be a Morley sequence in $p(x, a)$, and we want to prove that $q\left(x_{<\omega}, a\right)=$ $\operatorname{tp}\left(c_{<\omega} / a\right)$ does not divide over $a_{\sim}$. So let $\left(a_{i}: i<\omega\right)$ be an $a_{\sim}$-indiscernible sequence with $a_{0}=a$, and we need to prove that $\bigwedge_{i} q\left(x_{<\omega}, a_{i}\right)$ is consistent. 
We may extend the sequence by one element $a_{-1}$, and we may re-choose $c_{<\omega}$ such that $c_{<\omega} \downarrow_{a} a_{-1}$. As $\operatorname{tp}_{\text {next }}\left(c_{<\omega}\right) R p(x, a)$ and $a=a_{0} \sim a_{-1}$, we obtain $c_{<\omega} \downarrow_{a_{-1}} a$ by Lemma 3.9. As $\left(a_{i}: i<\omega\right)$ is $a_{-1}$-indiscernible, we can find $c_{<\omega}^{\prime}$ such that $c_{<\omega}^{\prime} a_{i} \equiv_{a^{\prime}} c_{<\omega} a$ for all $i<\omega$, so in particular $\vDash \bigwedge_{i} q\left(c_{<\omega}^{\prime}, a_{i}\right)$, as required.

(iii) $\Longrightarrow$ : If $p R p^{\prime}$ then clearly $p \sim p^{\prime}$. Taking $c_{<\omega}$ to witness $p R p^{\prime}$, then $a \downarrow_{c<\omega} a^{\prime} \Longrightarrow a \downarrow_{a \sim} a^{\prime} c_{<\omega}$.

$\Longleftarrow$ : We assume that $a \sim a^{\prime}$ and $a \downarrow_{a_{\sim}} a^{\prime}$. Let $c_{<\omega}$ be a Morley sequence in $p$ and $c_{<\omega} \downarrow_{a} a^{\prime}$, so:

$$
c_{<\omega} \underset{a}{\downarrow} a^{\prime} \Longrightarrow a c_{<\omega} \underset{a \sim}{\downarrow} a^{\prime} \Longrightarrow a \underset{c<\omega}{\downarrow} a^{\prime}
$$

Then $p(x, a) R \operatorname{tp}_{\text {next }}\left(c_{<\omega}\right) R p\left(x, a^{\prime}\right)$ by Lemma 3.9 , but then as $a \downarrow_{c_{<\omega}} a^{\prime}$ we can apply Lemma 3.8 to conclude that $p(x, a) R p\left(x, a^{\prime}\right)$.

(iv) Note $p_{0}=p \nmid a_{\sim}, p_{1}=p\left\lceil\operatorname{bdd}\left(a_{\sim}\right)\right.$. As $p$ is an amalgamation base, $p_{1}$ is a complete type, and in fact an amalgamation base. As $p \sim p_{1}$ they have the same parallelism class, whereby $\mathrm{Cb}\left(p_{1}\right)$ is interdefinable with $a_{\sim}$. Let $p_{2}$ be any extension of $p_{0}$ to $\operatorname{bdd}\left(a_{\sim}\right)$. Then it is an $a_{\sim}$-conjugate of $p_{1}$, whereby $\mathrm{Cb}\left(p_{1}\right)=\mathrm{Cb}\left(p_{2}\right)$ and $p_{1} \sim p_{2}$. We also have $\operatorname{bdd}\left(a_{\sim}\right) \downarrow_{a_{\sim}} \operatorname{bdd}\left(a_{\sim}\right)$, so $p_{1} R p_{2}$. In particular they have a common extension, whereby they are equal. Thus $p_{0}$ has a unique extension to $\operatorname{bdd}\left(a_{\sim}\right)$.

(v) Assume first that $b \subseteq a, p\lceil b$ is an amalgamation base, and $p$ does not divide over $b$. Then $p\left\lceil b \sim_{1} p, \operatorname{so} \operatorname{Cb}\left(p\lceil b)=a_{\sim}\right.\right.$ and in particular $a_{\sim} \subseteq b$. If we only know that $p$ does not divide over $b$, then consider $p\lceil\operatorname{bdd}(b)$ (this makes sense, as $p$ has a unique extension to $\operatorname{bdd}(a))$. Then $p \sim_{1} p \uparrow \operatorname{bdd}(b)$ and $a_{\sim} \subseteq \operatorname{bdd}(b)$. Conversely, if $a_{\sim} \subseteq \operatorname{bdd}(b)$, then $p$ does not divide over $\operatorname{bdd}(b)$ (as it does not divide over $a_{\sim}$ ), and therefore neither over $b$.

$\mathrm{QED}_{3.13}$

\section{EXAMPLES}

Example 4.1. The category $\mathscr{B}$ of Banach spaces is not simple.

Proof. The proof we give may not be the shortest one, but has the advantage of being a natural chain of deductions using the previous results. A direct counter-example to the local character may be deduced from it, of course. One should also mention that stronger results exist: Alex Usviatsov has shown that $\mathscr{B}$ has $S O P_{n}$ for every $n$, but this goes beyond our scope.

Assume that $\mathscr{B}$ is simple. Then we make the following observations:

- For sets $A, B, C$ in some big normed space, we define $A \oplus_{C}^{\mathscr{B}} B=$ $\operatorname{Span}(A C) \oplus_{\mathrm{Span}(C)}^{\mathscr{B}} \operatorname{Span}(B C)$. We say that $A$ and $B$ are direct summands over $C$ if $\operatorname{Span}(A B C)$ is isomorphic to $A \oplus_{C}^{\mathscr{B}} B$ over $A B C$. We claim that when this is true, $\operatorname{tp}(A / B C)$ does not divide over $C$ :

Indeed, let $\left\{B_{i}\right\}$ be any set of realizations of $\operatorname{tp}(B / C)$. Let $D=A \oplus_{C}^{\mathscr{B}} \cup B_{i}$. One verifies easily that for each $B_{i}$, the norm in $D$ on $\operatorname{Span}\left(A B_{i} C\right)$ is exactly 
the image of that on $A \oplus_{C}^{\mathscr{B}} B$, that is on $\operatorname{Span}(A B C)$ by assumption. Thus $\operatorname{tp}(A / B C)$ does not divide over $C$.

- Morley sequences exist in every type:

For any $B, C$, consider $\bigoplus_{i<\omega C}^{\mathscr{B}} B_{i}$, where $B_{i}$ are copies of $B$ over $C$. Then $\left(B_{i}\right)$ is an indiscernible sequence (in fact, an indiscernible set) over $C$, and each $B_{i}$ is a direct summand over $C$ with $B_{<i}$. By the previous claim, $\left(B_{i}\right)$ is a Morley sequence in $\operatorname{tp}(B / C)$.

- $A \downarrow_{C} B$ if and only if, whenever $b_{i} \in \operatorname{Span}(B C)$ for $i<n$ :

$$
\begin{array}{r}
\inf \left\{\sum\left\|b_{i}-a_{i}\right\|: a_{i} \in \operatorname{Span}(A C), \sum a_{i}=0\right\}= \\
\inf \left\{\sum\left\|b_{i}-c_{i}\right\|: c_{i} \in \operatorname{Span}(C), \sum c_{i}=0\right\}
\end{array}
$$

As $\mathscr{B}$ is simple, we know that $A \downarrow_{C} B$ if and only if for some Morley sequence $\left(B_{i}\right)$ in $\operatorname{tp}(B / C)$, we can realise $\operatorname{tp}(A / B)$ over all the $B_{i}$ simultaneously. In this, we may just as well use the Morley sequence given by the direct sum. So if it is true, we may suppose that $\operatorname{tp}\left(A B_{i} / C\right)=\operatorname{tp}(A B / C)$ for all $i$. Take $b_{i} \in \operatorname{Span}\left(B_{i} C\right)$ for $i<n$. Then by definition of the direct sum: $\left\|\sum b_{i}\right\|=$ $\inf \left\{\sum\left\|b_{i}-c_{i}\right\|: c_{i} \in \operatorname{Span}(C), \sum c_{i}=0\right\}$. On the other hand, by the triangle inequality: $\left\|\sum b_{i}\right\| \leq \inf \left\{\sum\left\|b_{i}-a_{i}\right\|: a_{i} \in \operatorname{Span}(A C), \sum a_{i}=0\right\}$. As the other inequality is clear, we obtain what we wanted. Conversely, this condition simply states that the natural mapping of $\bigoplus_{i<\omega C}{ }_{\mathscr{B}} B_{i}$ into $\bigoplus_{i<\omega A C}{ }^{\mathscr{B}} B_{i}$ is an isometry, and we thus obtain a copy of $A$ such that $\operatorname{tp}\left(A B_{i} / C\right)=\operatorname{tp}(A B / C)$ for all $i$. As it suffices to verify for a single Morley sequence, the independence is established.

- The above condition is asymmetric:

Consider a 2-dimensional vector space, with $a, b$ as a base. Consider the following norm: $\|\alpha a+\beta b\|=\max \{|\alpha|,|\beta-\alpha|\}$. Let $A=a, B=b, C=\varnothing$. Then for $\alpha_{i} a \in \operatorname{Span}(A)$ we have:

$$
\sum\left|\alpha_{i}\right|=\inf \left\{\sum \max \left\{\left|\alpha_{i}\right|,\left|\beta_{i}-\alpha_{i}\right|\right\}: \sum \beta_{i}=0\right\}
$$

But replacing $A$ and $B$ we have:

$$
\begin{aligned}
\|2 b\|+\|-2 b\| & =4 \\
& >2=\|2 b+a\|+\|-2 b-a\| \\
& \geq \inf \{\max \{|\alpha|,|2-\alpha|\}+\max \{|-\alpha|,|-2+\alpha|\}: \alpha \in \mathbb{F}\}
\end{aligned}
$$

As this is impossible in a simple cat, $\mathscr{B}$ is not simple.

$\mathrm{QED}_{4.1}$

Example 4.2. The class $\mathscr{H}$ of Hilbert space is stable, independence being orthogonality.

Example 4.3. Let $U l t$ be the category of ultrametric spaces, where the distances take values in $\omega$. Then $U l t$ is a compact abstract elementary category, whereby a cat, and is stable as such. However, types over $\varnothing$ are not extendible.

(We found this example in [BL03], where it was given rather in terms of equivalence relations, and attributed to Shelah). 
Proof. Verifying that Ult is an elementary category with amalgamation is easy and is left for the reader. One also sees that the type of a tuple is completely determined by the distances between elements of the tuple: this takes care of locality of types, so the only thing left to prove is weak compactness. By the description of types, we need to prove the following:

Claim. Let $G=(V, E, d)$ be a graph, where $V$ is the set of vertices and may be infinite, $E \subseteq[V]^{2}$ is the set of edges, and $d: E \rightarrow \omega$ is some map. Assume that every finite subgraph can be realised in $U l t$ where $d$ is the distance map: then $G$ can be realised in Ult.

Proof of claim. We may assume that $G$ is connected. For every path $v_{0}, v_{1}, \ldots, v_{n}$ in $G$, let $d_{0}(\bar{v})=\max _{i<n} d\left(v_{i}, v_{i+}\right)$, and for any $v, w \in V$ let $d_{1}(v, w)$ be the minimum of $d_{0}(\bar{v})$ where $\bar{v}$ is a path connecting $v$ and $w$.

If $\{v, w\} \in E$, then $d_{1}(v, w)=d(v, w): d_{1}(v, w) \leq d(v, w)$ by definition, and if the inequality is strict we get a contradiction to finite realisability. On the other hand, it follows from the construction that $\left(V, d_{1}\right) \in U l t$, so $G$ is realised in Ult. $\quad$ QED $_{\text {Claim }}$

In order to see that $U l t$ is stable, we observe that $\operatorname{tp}(a / A)$ is completely determined by $d(a, A)$ and by an element $b \in A$ such that $d(a, b)=d(a, A)$ : counting types follows. Finally, whatever be $a$ and $b, \operatorname{tp}(a / b)$ divides over $\varnothing$ : Let $n=d(a, b)$, so $\operatorname{tp}(a / b)$ just says $d(x, b)=n$, and take an indiscernible sequence $\left(b_{i}\right)$ such that $d\left(b_{0}, b_{1}\right)>n$, so $\bigwedge d\left(x, b_{i}\right)=n$ is inconsistent.

$\mathrm{QED}_{4.3}$

\section{REFERENCES}

[Bena] Itay Ben-Yaacov, Thickness, and a categoric view of type-space functors, Fundamenta Mathematicae (to appear).

[Benb] Uncountable dense categoricity in cats, preprint.

[Ben03] _ Positive model theory and compact abstract theories, Journal of Mathematical Logic 3 (2003), no. 1, 85-118.

[BL03] Steven Buechler and Olivier Lessmann, Simple homogeneous models, Journal of the American Mathematical Society 16 (2003), 91-121.

[HKP00] Bradd Hart, Byunghan Kim, and Anand Pillay, Coordinatisation and canonical bases in simple theories, Journal of Symbolic Logic 65 (2000), 293-309.

[Kim98] Byunghan Kim, Forking in simple unstable theories, Journal of the London Mathematical Society 57 (1998), no. 2, 257-267.

[KP97] Byunghan Kim and Anand Pillay, Simple theories, Annals of Pure and Applied Logic 88 (1997), 149-164.

[Pil98] Anand Pillay, Definability and definable groups in simple theories, Journal of Symbolic Logic 63 (1998), 788-796.

[Pil00] _ Forking in the category of existentially closed structures, Connections between Model Theory and Algebraic and Analytic Geometry (Angus Macintyre, ed.), Quaderni di Matematica, vol. 6, University of Naples, 2000.

[Sha00] Ziv Shami, Definability in low simple theories, Journal of Symbolic Logic 65 (2000), no. 4, 1481-1490.

[She75] Saharon Shelah, The lazy model-theoretician's guide to stability, Logique et Analyse 71-72 (1975), 241-308. 
Itay Ben-Yaacov, Massachusetts Institute of Technology, Department of Mathematics, 77 Massachusetts Avenue, Room 2-101, Cambridge, MA 02139-4307, USA

E-mail address: pezz@math.mit.edu

$U R L:$ http://www-math.mit.edu/ pezz 